\title{
Diatomeas (Bacillariophyceae) subfósiles del Brazo Blest del Lago Nahuel Huapi, Argentina
}

\author{
M. LUJÁN GARCÍA ${ }^{1}$ y NORA I. MAIDANA ${ }^{1}$
}

\begin{abstract}
Summary: Subfossil diatoms from Lago Nahuel Huapi (Brazo Blest), Argentina. The main objective of this study was to analyze subfossil diatoms from Nahuel Huapi Lake (Brazo Blest) in order to increase the knowledge about the region's phycoflora, which until nowadays has been poorly studied. There were 47 genera and 114 infrageneric taxa recorded, from which 1 is recorded for first time for Argentina and 18 are new records for Northern Patagonia (Argentina).
\end{abstract}

Key words: Biodiversity, phycology, Patagonia, lakes.

Resumen: El objetivo principal de este trabajo fue estudiar las diatomeas subfósiles del Brazo Blest del Lago Nahuel Huapi y así aportar información sobre la ficoflora de la región, la cual hasta hoy ha sido poco estudiada. Se registraron 47 géneros y 114 taxones infragenéricos, de los cuales uno es un nuevo registro para Argentina y 18 son nuevos para el Norte de la Patagonia (Argentina).

Palabras clave: Biodiversidad, ficología, Patagonia, lagos.

\section{INTRODUCCIÓN}

Las algas más que ningún otro organismo, son utilizadas para inferir condiciones ambientales pasadas de un cuerpo de agua a través de la identificación de las especies y sus preferencias ecológicas (Maidana \& Amenabar, 2014). Las diatomeas (Bacillariophyceae) constituyen un importante componente de las comunidades acuáticas, tienen una distribución cosmopolita y ocupan una amplia variedad de hábitats ya que su distribución está relacionada con la química del agua, el clima y la geología (Battarbee, 1986; Round et al., 1990). Poseen una gran variedad de estrategias adaptativas y su corto ciclo de vida les permite responder rápidamente a los cambios ambientales por lo que son uno de los indicadores biológicos más utilizados (Blanco et al., 2004; Urrea \& Sabater, 2009).

\footnotetext{
1 IBBEA (CONICET - UBA); DBBE - FCEyN - UBA. Laboratorio de Diatomeas Continentales. Int. Güiraldes 2160, Ciudad Universitaria, Pabellón 2, piso 4, Lab 15. E-mail: mlgarcia@bg.fcen.uba.ar
}

La región andino-patagónica contiene numerosos lagos que le aportan un gran atractivo turístico que atrae visitantes de todas partes del mundo, favoreciendo la instalación de hoteles, centros de pesca y de esquí, grandes y pequeñas embarcaciones, etc.

En particular, en la cuenca del Lago Nahuel Huapi, ubicado en el Parque Nacional del mismo nombre, estas actividades han crecido en los últimos 100 años no siempre en forma amigable con el medio ambiente, lo que podría estar produciendo cambios en el lago y otros cuerpos de agua de la región, con el consecuente deterioro de la calidad del agua. Es por ello que se hace imprescindible disponer de una línea de base del conocimiento de la biodiversidad local a fin de poder registrar en el futuro el posible impacto negativo de la actividad antrópica en el área y poder planear un control adecuado si así lo requiriese.

Las distintas especies de diatomeas constituyen una valiosa herramienta para realizar inferencias paleoambientales (Lotter et al., 1999; Recasens et al., 2015) por lo que el conocimiento de la composición de los ensambles recuperados de secuencias sedimentarias de lagos y lagunas puede 
aportar valiosa información para comprender cambios en el ambiente, ya sean naturales o producidos por la actividad humana en la región.

Los estudios previos sobre la ficoflora de la provincia de Río Negro son pocos pero deben destacarse los aportes de Krasske (1949) referidos a ambientes lacustres cuaternarios de nuestro país y los de Frenguelli (1924, 1942; Frenguelli \& Orlando, 1956, entre otros), pionero de los estudios diatomológicos, tanto fósiles como actuales, cubriendo gran parte del territorio argentino e incluso el Sector Antártico e Islas del Atlántico Sur. Otros trabajos sobre algas en la región que deben ser mencionados son los de Izaguirre et al. (1990), Izaguirre \& Saad (2014), Maidana (1996), Maidana et al. (2005), Tell et al. (2011) y Thomasson (1959, 1963).

Como parte del proyecto interdisciplinario "Estudios paleoambientales en sedimentos lacustres: El fenómeno del ENSO y los cambios climáticos ocurridos en el ultimo milenio en Patagonia Norte" (CONICET. PIP 112-20080102345) se estudiaron los ensambles de diatomeas contenidos de un testigo sedimentario recuperado en 2011 y que cubre los últimos 100 años de la historia del lago. Particularmente, en esta contribución se presenta la composición de los ensambles de diatomeas registradas para este testigo con el fin de incrementar los datos sobre la biodiversidad diatomológica para el Lago Nahuel Huapi.

\section{MATERIALES Y MÉTOdos}

El Lago Nahuel Huapi está situado en el Parque Nacional con el mismo nombre (740-767 m snm); y forma parte del límite entre las provincias argentinas de Río Negro y Neuquén. Biogeográficamente, se ubica en la Provincia de la Patagonia Central, Subregión Patagónica, de la Región Andina, según la clasificación de Morrone (2001). El lago tiene una forma ameboidal, con brazos orientados en todas direcciones. Tiene una profundidad máxima de 438 $\mathrm{m}$ y aproximadamente $557 \mathrm{~km}^{2}$ de superficie, con un gran desarrollo de la línea de costa $(357 \mathrm{~km})$ debido a la forma indentada de la costa, con numerosas bahías y playas. En su totalidad, el lago está rodeado por montañas con abundante vegetación, principalmente bosques de Austrocedrus y Nothofagus. El Brazo

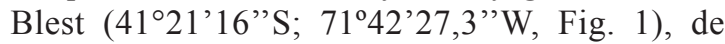
donde provienen las muestras analizadas en este trabajo, está ubicado en el lado oeste del lago. Particularmente, en la bahía que forma este brazo se descargan las aguas del lago Frías a través del río del mismo nombre, el que a su vez está alimentado por el deshielo del glaciar Tronador.

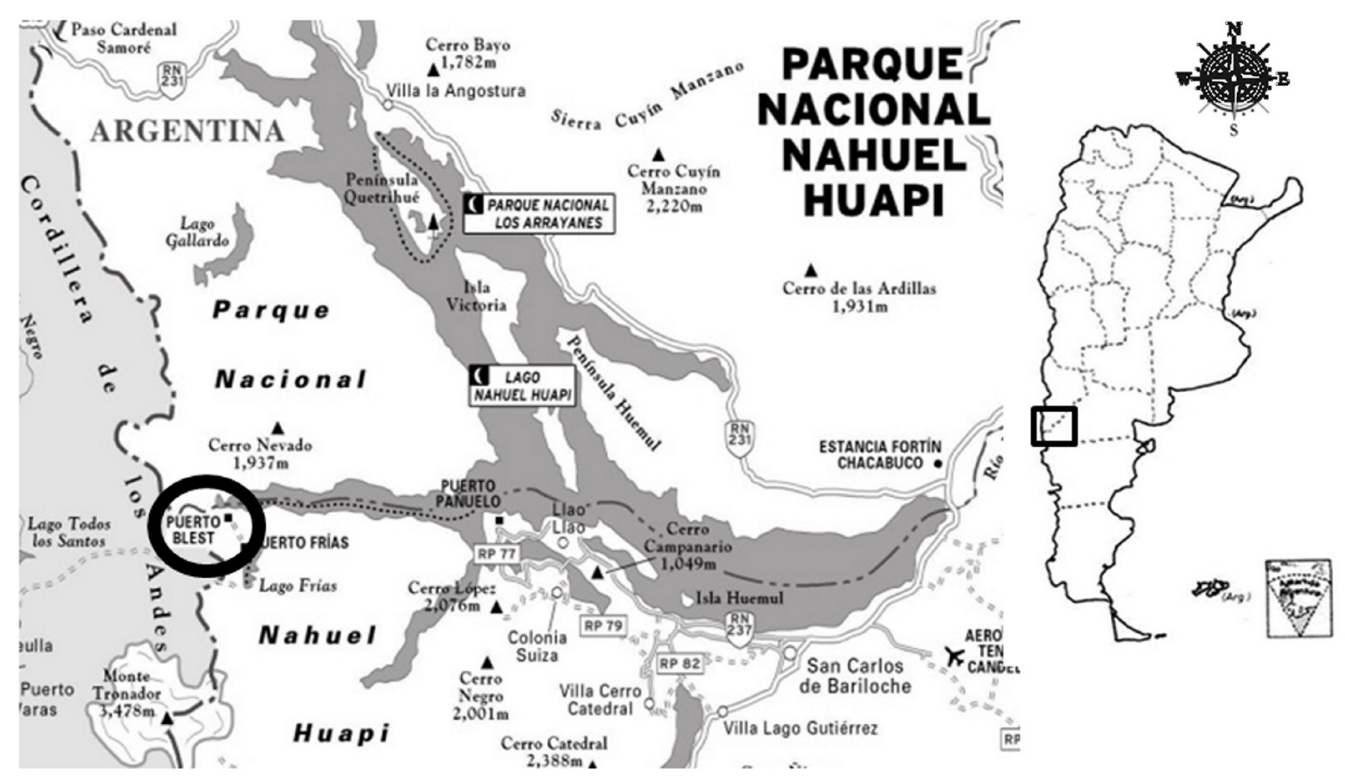

Fig. 1. Ubicación del sitio de muestreo del testigo sedimentario analizado. 


\section{L. García y N. I. Maidana - Diatomeas subfósiles del Brazo Blest, Argentina}

El material estudiado proviene del testigo sedimentario Blest11 obtenido a $12 \mathrm{~m}$ de profundidad del fondo del Brazo Blest, con un muestreador tipo Hammer Corer. Los primeros $35 \mathrm{~cm}$ se muestrearon cada 1-2 cm, obteniéndose 27 muestras. Una submuestra de cada nivel se destinó para el análisis diatomológico y otras para análisis geoquímicos y sedimentológicos.

El análisis diatomológico se realizó siguiendo las técnicas estándar para el estudio cualitativo y cuantitativo (número de valvas por gramo de sedimento seco). Para esto se tomó una fracción de cada muestra, se la secó en estufa a $80^{\circ} \mathrm{C}$ y luego se la pesó. Posteriormente, se eliminó todo resto de materia orgánica que impidiera la correcta observación de los caracteres diagnósticos, oxidando con $\mathrm{H}_{2} \mathrm{O}_{2}(100 \mathrm{Vol})$ en caliente (Battarbee, 1986). Luego de remover los restos de los reactivos mediante sucesivos lavados con agua destilada, se agregó a la suspensión resultante un volumen conocido de microsferas de poliestireno para estimar las abundancias absolutas (Battarbee \& Kneen, 1982). Los preparados permanentes se confeccionaron con resina Naphrax ${ }^{\circledR}$ como medio de montaje y se depositaron en la colección del Laboratorio de Diatomeas Continentales (Departamento de Biodiversidad y Biología Experimental, Facultad de Ciencias Exactas y Naturales, Universidad de Buenos Aires).

Las observaciones se realizaron con un microscopio óptico binocular (MO) Polivar Reichert-Jung, equipado con cámara fotográfica automática digital con la que se registraron todos los taxones hallados. Los individuos de difícil identificación se observaron con el microscopio electrónico de barrido (MEB) Carl Zeiss SUPRA40 del Centro de Microscopía Avanzada de la Facultad de Ciencias Exactas y Naturales (UBA).

Para la identificación taxonómica se tuvo en cuenta el rango de variación de rasgos morfológicos y morfométricos. Las abreviaturas utilizadas en las descripciones son: VR (valva con rafe), VSR (valva sin rafe) y EA/ET (eje apical/eje transapical).

La sinonimia fue tomada, fundamentalmente, de VanLandingham (1967-1979) y de trabajos específicos posteriores (incluyendo http://www.algaebase.org). La distribución geográfica de los taxones en Argentina fue consultada en Vouilloud (2003).

En cada preparado, se contó un mínimo de 400 valvas para estimar las abundancias relativas de los taxones en cada muestra.

\section{Resultados}

En esta contribución se presenta la lista de 47 géneros y 114 taxones infragenéricos (Tabla 1), que se reconocieron en las 27 muestras analizadas a lo largo de los $35 \mathrm{~cm}$ del testigo sedimentario analizado en este estudio.

De los 114 taxones infragenéricos identificados, uno se menciona por primera vez para Argentina y 18 son nuevos registros para la provincia de Río Negro. En el testigo sedimentario analizado, los géneros mejor representados considerando el número de especies fueron Pinnularia (14), Gomphonema (7), Navicula (7), Nitzschia (6), Planothidium (6), Stauroneis (5), Eunotia (5) y Achnanthidium (5). Cuatro taxones (3,5\% del total) no pudieron ser identificados a nivel específico.

Seencontraron especies aparentementeendémicas de Sudamérica como Cymbopleura tsoneka, Encyonema jemtlandicum var. venezolana y Gomphonema punae y otras características de ambientes fríos como Psammothidium abundans, Brachysira microcephala y Gomphonema aff. possessionense. Sin embargo, la mayoría de las especies identificadas están consideradas como cosmopolitas.

Aulacoseira distans, A. granulata, Discostella spp, Urosolenia eriensis, Achnanthidium minutissimum, A. subatomoides, Cavinula pseudocutiformis, Encyonopsis microcepahala, Eolimna minima, Eunotia bilunaris, E. implicata, Gomphonema gracile, Sellaphora pupula, Tabellaria flocculosa y las pequeñas fragilarioides representan el 12,4\% del total de taxones identificados y fueron las especies más abundantes en cuanto a número de individuos en toda la secuencia estudiada (Tabla 2) mientras que las restantes estuvieron escasamente representadas.

En todas las muestras predominaron las Fragilariaceae, representadas por especies de los géneros Staurosira, Staurosirella, Punctastriata, Pseudostaurosira y Fragilaria y, en algunos casos, co-dominó Discostella stelligera. La mayoría de las Fragilariaceae halladas en el Brazo Blest tienen un tamaño muy pequeño, por lo que fue necesaria la ayuda del microscopio electrónico de barrido para su identificación y no fue posible estimar sus abundancias relativas individuales.

A continuación se presentan los taxones que se citan por primera vez para la región o para el país y/o que resultan interesantes por sus características morfológicas: 
Achnanthidium catenatum (Bílý \& Marvan) Lange-Bertalot. Fig. 2: A; Fig. 3: A-B.

Hlúbiková et al. 2011: 23-27, Figs. 1-34; 148155; Krammer \& Lange-Bertalot 2004: 62, Fig. 34: 23, 24 (como Achnanthes catenata).

Valvas delgadas con terminaciones, en general capitadas, subcapitadas en los ejemplares más pequeños. Rafe filiforme, con las fisuras proximales algo distanciadas y las distales no prolongadas más allá de la helictoglosa. En ambas valvas el área axial es lineal y la central pequeña o ausente. En vista cingular, los ápices de ambas valvas están fuertemente curvados hacia la VSR, mientras que la porción media del frústulo es recta o ligeramente ondulada. Estrías radiales en ambas valvas, compuestas por areolas circulares; las 3-4 estrías centrales están algo más distanciadas entre sí que las restantes. Manto valvar con una única hilera de areolas transversalmente elongadas.

VR: Eje apical 17,1-26,7 $\mu \mathrm{m}$; eje transapical 2,24,6 $\mu \mathrm{m}$; estrías 24-38 en $10 \mu \mathrm{m}$.

Obs.: La longitud del eje apical de algunos de los ejemplares observados es mayor que la indicada para el tipo $(10-17,5 \mu \mathrm{m})$ por Hlúbiková et al. (2011).

Adlafia minuscula (Grunow) Lange-Bertalot. Fig. 3: D.

Lange-Bertalot 2001: 143; Fig. 108: 4-10; Fig. 106: 5-8.

Valvas elíptico-lanceoladas, extremos redondeados levemente subrostrados. Rafe filiforme, recto, extremos centrales simples; extremos terminales curvados. Área axial estrecha, lineal; sin área central. Estrías radiales en el área central y convergentes hacia los ápices.

Eje apical 9,8 $\mu \mathrm{m}$; eje transapical 2,9 $\mu \mathrm{m}$; estrías 40 en $10 \mu \mathrm{m}$.

\section{Brachysira microcephala (Gunow) Compère Fig.} 2: B; Fig. 3: C.

Wolfe \& Kling 2001: 250-252, Figs. 14-22; Rumrich et al. 2000: 418 (como Brachysira neoexilis Lange-Bertatolt \& Moser)

Valvas rómbico-lanceoladas con ápices ligeramente subrostrados. Área axial angosta, lineal; área central pequeña, redondeada. Rafe filiforme, recto. Estrías transapicales radiales a subparalelas en los ápices, difíciles de distinguir al MO. Superficie valvar a veces cubierta por espinas diminutas, solo distinguibles con MEB.

Eje apical 21,9-28,8 $\mu \mathrm{m}$; eje transapical 3,9-4,4 $\mu \mathrm{m}$; estrías 29-36 en $10 \mu \mathrm{m}$.

Obs.: Según Wolfe \& Kling (2001), LangeBertatolt \& Moser (1994) asignaron el nombre de $B$. neoexilis a la misma especie que había sido previamente transferida por Compère en 1988. Werum \& Lange-Bertalot (2004) describen $B$. neglectissima, diferenciándola de $B$. neoexilis solo por la densidad de estrías ligeramente mayor (36-40 y no 30-36 estrías en $10 \mu \mathrm{m}$ ) y por la disposición de las estrías en los extremos, desde moderadamente radiales a subparalelas.

Cavinula cocconeiformis f. elliptica (Hustedt) Lange-Bertalot. Fig. 2: C; Fig. 3: E.

Krammer \& Lange-Bertalot 1986: 158; Fig. 59: 2-5 (como Navicula cocconeiformis Gregory ex Greville).

Valvas anchamente lanceoladas con extremos ligeramente subrostrados y ápices cuneadoredondeados. Área axial angostamente lanceolada; área central ausente. Rafe filiforme; los extremos distales se alojan en un área hialina próxima al ápice y los proximales terminan en una depresión poco profunda. Estrías con areolas delicadas, radiales en toda la valva, con algunas estrías más cortas intercaladas en la zona media de la valva.

Eje apical 12,1-16,4 $\mu \mathrm{m}$; eje transapical 6,4-9,5 $\mu \mathrm{m}$; estrías $20-32$ en $10 \mu \mathrm{m}$; areolas $34-40$ en 10 $\mu \mathrm{m}$.

Craticula halophila (Grunow) Mann. Fig. 2: D.

Lange-Bertalot 2001: 114, Fig. 89: 1-7.

Valva lanceolada con extremos levemente proyectados. Área axial angosta, lineal; sin área central. Rafe recto con poros centrales poco evidentes al MO. Las estrías están dispuestas de forma radial.

Eje apical $72,5 \mu \mathrm{m}$; eje transapical $14,6 \mu \mathrm{m}$; estrías 15 en $10 \mu \mathrm{m}$.

Encyonema neogracile Krammer. Fig. 2: E-F.

Krammer 1997a: 142. Fig. 82: 1-13; Fig. 83: 1-7; Fig. 85: 1-12; Fig. 91: 1, 2; Fig. 93: 1; Fig. 97: 3, 6.

Valvas semi-lanceoladas, con el lado ventral levemente convexo, casi recto. Extremos angostos, rostrados, moderadamente curvados hacia el lado ventral, sin formar un "hombro" en la parte dorsal. Área axial angosta, lineal; área central ausente. 
M. L. García y N. I. Maidana - Diatomeas subfósiles del Brazo Blest, Argentina
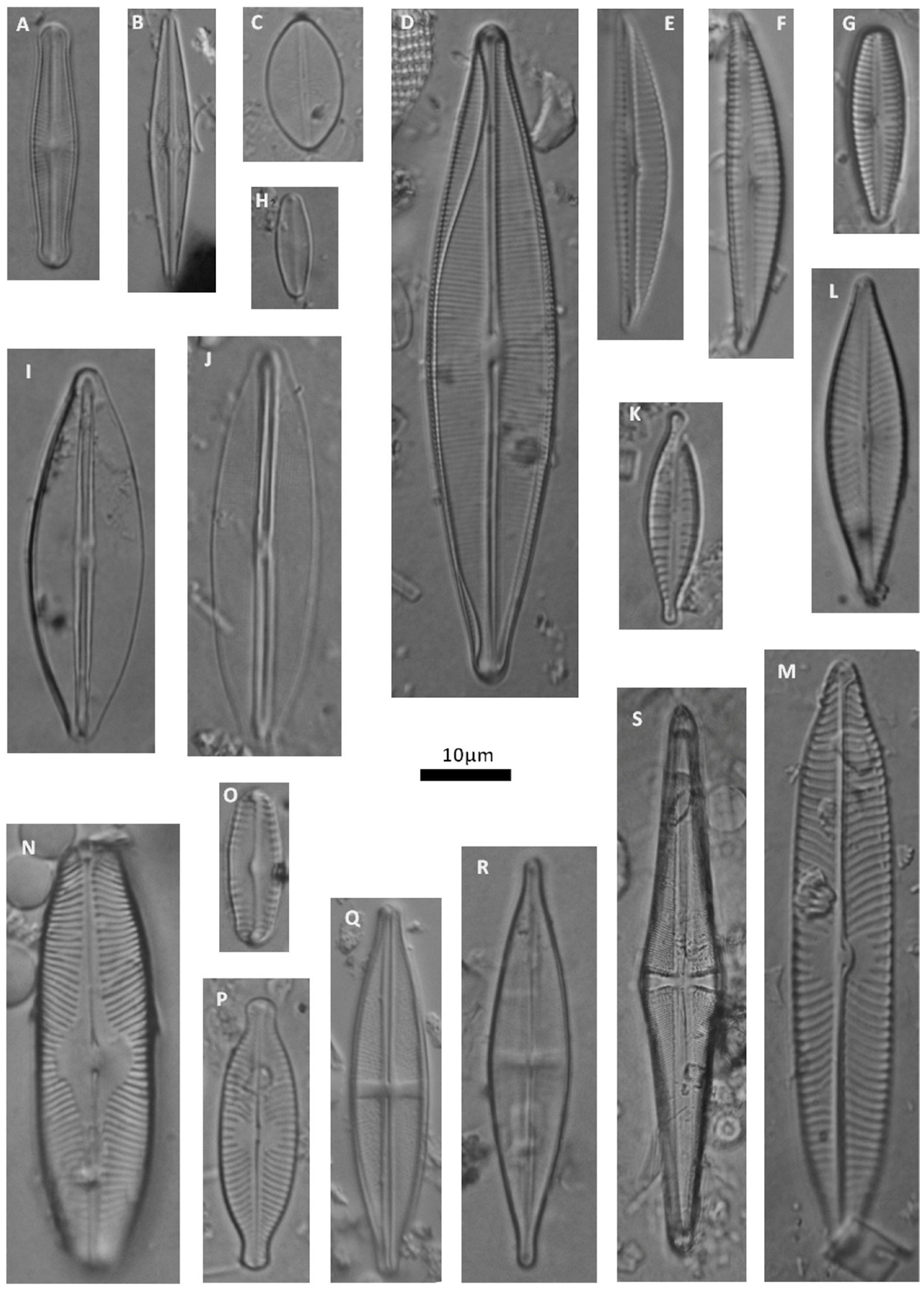

Fig. 2. A: Achnanthidium catenatum. B: Brachysira microcephala. C: Cavinula cocconeiformis f. elliptica. D: Craticula halophila. E-F: Encyonema neogracile. G: Gomphonema punae. H: Eolimna minima. I-J: Frustulia saxonica. K: Gomphonema aff. possessionense. L: Navicula trivialis. M: N. neomundana. N: Pinnularia aff. renata. O: Pinnularia sp. P: Placoneis paraelginensis. Q: Stauroneis aff. delicata. R: S. aff. vandervijveri. S: S. actua. A-S Escala $=10 \mu \mathrm{m}$. 
Bol. Soc. Argent. Bot. 50 (2) 2015
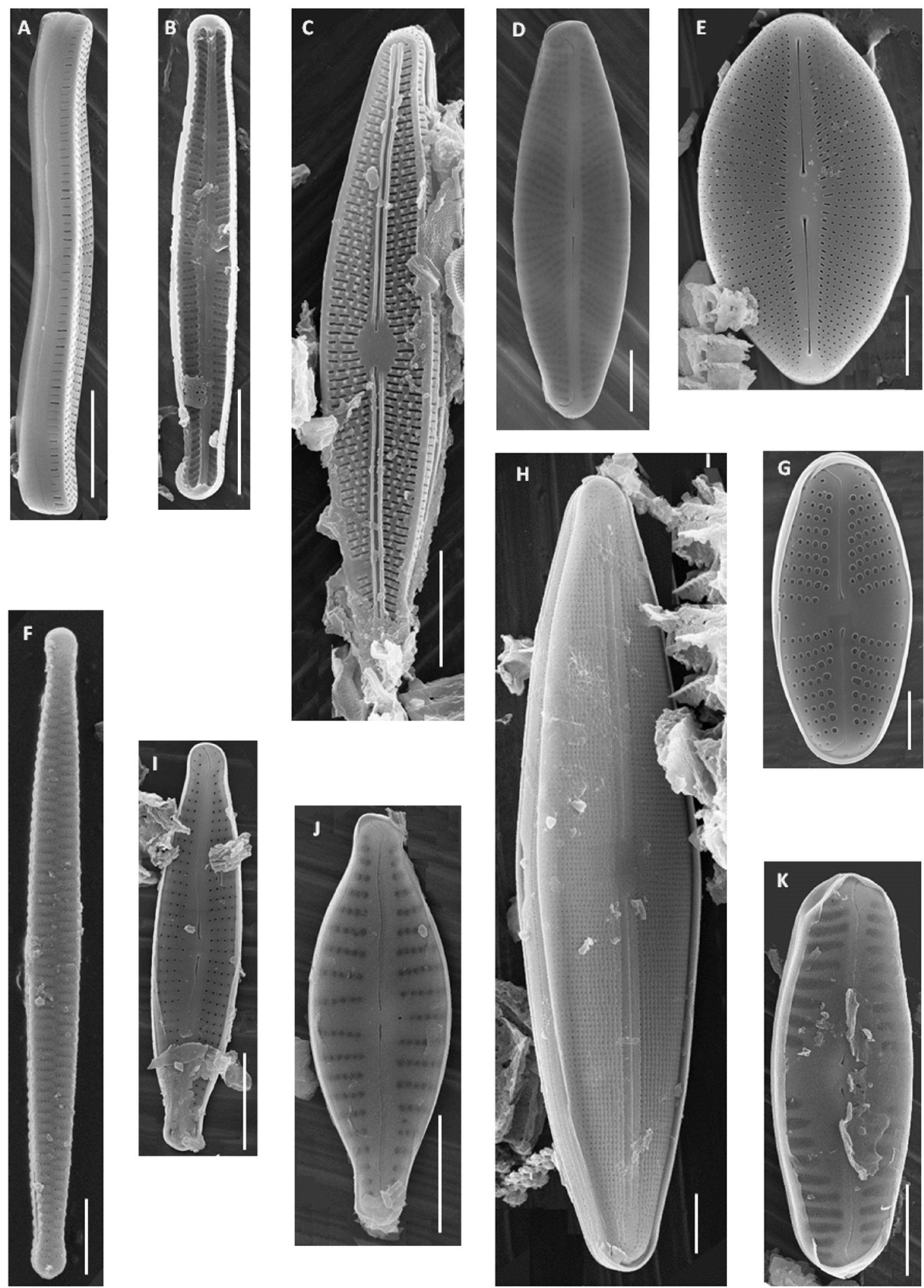

Fig. 3. A-B: Achnanthidium catenatum. C: Brachysira microcephala. D: Adlafia minuscula. E: Cavinula cocconeiformis f. elliptica. F: Fragilaria famelica. G: Eolimna minima. H: Frustulia saxonica. I: Encyonopsis subminuta. J: Gomphonema aff. possessionense. K: Pinnularia sp. Escala $=5 \mu \mathrm{m}$; excepto D y G Escala $=2 \mu \mathrm{m}$. 


\section{L. García y N. I. Maidana - Diatomeas subfósiles del Brazo Blest, Argentina}

Los extremos distales son largos y están curvados hacia el lado ventral. El estigmoide ventral es casi indistinguible $\mathrm{o}$ ausente.

Eje apical 34,2-36,7 $\mu \mathrm{m}$; eje transapical 5,7-5,9 $\mu \mathrm{m}$; relación EA/ET 5,9-6,1; estrías 13-14 en $10 \mu \mathrm{m}$.

Encyonopsis subminuta Krammer \& Reichardt. Fig. 3: I.

Krammer 1997b: 96; Fig. 143a: 30-33; Fig. 144: 1-11, 16, 17; Fig. 149: 11-16; Fig. 150: 15-21.

Valvas angostamente lanceoladas, levemente dorsiventrales. Extremos capitados, no claramente separados del cuerpo principal de la valva por la presencia de "hombros". Área axial estrecha, lineal; área central ausente. Rafe filiforme, fisuras externas curvadas hacia el lado ventral. Extremos proximales curvados hacia el lado dorsal, extremos terminales hacia el lado ventral.

Eje apical 15,4 $\mu \mathrm{m}$; eje transapical $3,2 \mu \mathrm{m}$; relación EA/ET 4,8; estrías 28 en $10 \mu \mathrm{m}$.

Obs.: Esta especie es frecuentemente confundida con E. microcephala (Grunow) Krammer, de la cual se diferencia por la ausencia de "hombros" que marquen una separación entre los extremos y el cuerpo principal de la valva (comparar la fig. 144: 1-11 con la fig. 149: 1-8 en Krammer, 1997b).

Eolimna minima (Grunow) Lange-Bertalot. Fig. 2: H; Fig. 3: G.

Werum \& Lange-Bertalot 2004: 79, Fig. 40: 2. Blanco et al. 2010: 86; Fig. 48: 58-84; Fig. 53: 1-3.

Valvas elípticas con extremos redondeados. Área axial angosta, lineal; área central transversal, de tamaño variable, asimétrica, formada por el acortamiento de 1-2 estrías centrales que se encuentran más distanciadas que las restantes. Rafe filiforme, recto, con los poros centrales pequeños y curvados hacia el lado primario de la valva; las fisuras distales son largas y están fuertemente curvadas hacia el lado secundario. Estrías radiales en toda la valva, con areolas redondeadas a cuadrangulares, de tamaño creciente desde el margen hacia el centro.

Eje apical 6,7-11,5 $\mu \mathrm{m}$; eje transapical 3-3,5 $\mu \mathrm{m}$; estrías 33 en $10 \mu \mathrm{m}$; areolas 55 en $10 \mu \mathrm{m}$.

Fragilaria famelica (Kützing) Lange-Bertalot. Fig. 3: F.

Krammer \& Lange-Bertalot 1991: 128, Fig. 111: 4-12, 16, 17.

Valvas muy angostamente lanceoladas, con extremos subcapitados. Área axial muy angosta, sólo distinguible con MEB; área central ausente. Estrías paralelas, delicadas, opuestas; areolas circulares. Campos porosos apicales poco diferenciados. Rimopórtula presente en uno de los ápices.

Eje apical 31,7-46,2 $\mu \mathrm{m}$; eje transapical 1,7-2,5 $\mu \mathrm{m}$; estrías 22-23 en $10 \mu \mathrm{m}$.

Frustulia saxonica Rabenhorst. Fig. 2: I-J; Fig. 3: $\mathrm{H}$.

Werum \& Lange-Bertalot 2004: Fig. 51: 4-6.

Valvas lanceoladas a rómbico-lanceoladas, con extremos subrostrados. Área axial estrecha, lineal; área central ausente. Costillas axiales constrictas a la altura del nódulo central. Las estrías, no siempre distinguibles con $\mathrm{MO}$, son paralelas en toda la valva.

Eje apical 42,5-67,1 $\mu \mathrm{m}$; eje transapical 10,8$14,5 \mu \mathrm{m}$; estrías 28-39 en $10 \mu \mathrm{m}$.

Gomphonema punae Lange-Bertalot \& Rumrich. Fig. 2: G.

Rumrich et al. 2000: 140, Fig. 129: 1-14. Sala et al. 2008: 1170, Fig. 34-36.

Valvas claviformes con extremos subrostrados y ápices truncado-redondeados. Área axial angosta, lineal; área central unilateral, delimitada por el acortamiento de una estría central. Un estigmoide en el extremo de la estría central más larga. Estrías levemente radiales. Areolas difíciles de distinguir con MO.

Eje apical 21,1-28,8 $\mu \mathrm{m}$; eje transapical 5,8-6,8 $\mu \mathrm{m}$; estrías $10-15$ en $10 \mu \mathrm{m}$.

Gomphonema aff. possessionense Van de Vijver \& Beyens. Fig. 2: K; Fig. 3: J.

Van de Vijver et al. 2002: 51, Fig. 81: 24-32.

Valvas cuneado-lanceoladas, poco heteropolares, con ápices rostrado-capitados a capitados. Área axial angosta, lineal; área central asimétrica debido al acortamiento de una de las estrías centrales, que puede llegar a faltar. Estigmoide próximo a la estría central más larga. Rafe recto, filiforme con poros centrales poco marcados. Estrías subparalelas. Areolas no distinguibles con MO.

Eje apical 18,5-22,2 $\mu \mathrm{m}$; eje transapical 4,5-5,5 $\mu \mathrm{m}$; relación EA/ET 4,05-4,06 estrías $11-18$ en 10 $\mu \mathrm{m}$.

Obs.: Los dos ejemplares observados difieren de $G$. possessionense fundamentalmente por su 
relación EA/ET ligeramente menor (4,87-5,61, según las figuras 81: 24-32 de Van de Vijver et al., 2002). Consideramos necesaria la observación de un mayor número de valvas para la correcta identificación de este material.

Navicula neomundana (Lange-Bertalot \& Rumrich) Lange-Bertalot, Jarlman \& Van de Vijver. Fig. 2: M.

Van de Vijver \& Lange-Bertalot 2009: 424, Fig. 87-95; Rumrich et al. 2000: 175, Fig. 38: 1-4; Fig. 37: 5-8 (como Navicula viridulacalcis spp. neomundana).

Valvas lineales a lineal-lanceoladas con extremos subrostrados. Área axial angosta, lineal; área central transversalmente redondeada, algo asimétrica, formada por el acortamiento regular de 4 a 5 estrías centrales. Rafe filiforme, con las fisuras proximales levemente flexionadas hacia el lado primario de la valva. Estrías radiales en casi toda la valva y convergentes hacia los ápices.

Eje apical 53,8-65,4 $\mu \mathrm{m}$; eje transapical 10-10,9 $\mu \mathrm{m}$; estrías 9 en $10 \mu \mathrm{m}$.

Navicula trivialis Lange-Bertalot. Fig. 2: L.

Lange-Bertalot 2001: 73, Fig. 29: 1-6; 64: 1; 68: 1, 2. Blanco et al. 2010: 82, Fig. 58: 42-49.

Valvas lanceoladas con extremos subrostrados, angostos. Área axial angosta, lineal; área central transversalmente redondeada, ligeramente asimétrica por el acortamiento irregular de 3-4 estrías a ambos lados del nódulo central. Rafe filiforme, recto. Estrías radiales en casi toda la valva, tornándose subparalelas muy cerca de los extremos.

Eje apical 35,5-44,7 $\mu \mathrm{m}$; eje transapical 8,9-10,7 $\mu \mathrm{m}$; estrías 9-12 en $10 \mu \mathrm{m}$.

\section{Pinnularia aff. renata Krammer. Fig. 2: N.}

Krammer 2000: 79, Fig. 59: 1-7.

Valvas anchas, lineares, con extremos subrostrados y ápices truncado-redondeados. Rafe lateral, con las fisuras proximales externas débilmente curvadas hacia el lado primario; poros centrales en forma de gota. Alveolos radiales en la zona central de la valva, luego paralelos y convergentes en los extremos. Área axial angostamente lanceolada, ensanchándose hacia el área central, la cual es orbicular y algo asimétrica.

Eje apical 44,8 $\mu \mathrm{m}$; eje transapical 11,7 $\mu \mathrm{m}$; relación EA/ET 3,83; estrías 13 en $10 \mu \mathrm{m}$.

Obs.: Este material se asemeja a $P$. renata Krammer, en la forma general de la valva y en la forma y amplitud de las áreas axial y central. Sin embargo, el ancho valvar y la densidad de estrías son ligeramente mayores. En el material estudiado solo hemos encontrado 2 valvas que, por sus dimensiones iguales, podrían pertenecer a un mismo individuo. Es necesario analizar un mayor número de valvas para poder identificar este taxón con mayor precisión

\section{Pinnularia sp. Fig. 2: O; Fig. 3: K.}

Valvas lineales a elípticas, con extremos subrostrados. Rafe lateral, con las fisuras distales externas levemente curvadas en forma de hoz hacia el lado secundario de la valva; poros centrales marcados, curvados hacia el lado primario. Área axial estrecha, ensanchándose hacia el área central, área central, la que forma una fascia amplia. Alveolos paralelos a ligeramente radiales en el centro de la valva, paralelos a ligeramente convergentes en los extremos.

Eje apical 16,3-17 $\mu \mathrm{m}$; eje transapical 5,5-5,7 $\mu \mathrm{m}$; estrías 13-14 en $10 \mu \mathrm{m}$.

Obs.: Hemos hallado solo 3 ejemplares de este taxón $\mathrm{y}$, si bien guarda cierta semejanza con $P$. metzeltinii Krammer, hay diferencias en cuanto a la forma general de la valva y sus extremos. Consideramos necesaria la observación de un mayor número de valvas para la correcta identificación de este material.

Placoneis paraelginensis Lange-Bertalot. Fig. 2: P. Rumrich et al. 2000: 208, Fig. 60: 17-20; Cox 2003: Fig. 63-65, 70, 71.

Valvas lineal-lanceoladas, con extremos subcapitados. Área axial angosta, lineal; área central transversal, delimitada por el acortamiento de 4 estrías centrales. Rafe filiforme con las fisuras proximales curvadas en el mismo sentido. Estrías radiales en toda la valva. Areolas no distinguibles con MO. Estigmoide ausente.

Eje apical 28,1 $\mu \mathrm{m}$; eje transapical $8,1 \mu \mathrm{m}$; relación EA/ET 3,48; ancho de los ápices $2,9 \mu \mathrm{m}$, estrías 16 en $10 \mu \mathrm{m}$.

Obs.: P. paraelginensis se diferencia de $P$. elginensis (Gregory) Cox fundamentalmente por tener valvas lineales, en vez de elípticas. Cox (2003) señala que Rumrich et al. (2000) incluyeron 
en el material que ilustran como $P$. paraelginensis (Fig. 60: 17-20) a, por lo menos, 3 especies diferentes de las cuales solo una de ellas (Fig. 60: 18) correspondería a P. paraelginensis y sugiere que solo las formas elípticas que aparecen ilustradas en Krammer \& Lange-Bertalot (1986: Fig. 46:1-6) serían realmente $P$. elginensis.

\section{Psammothidium abundans (Manguin)}

Bukhtiyarova \& Round. Fig. 4: B-C.

Van de Vijver et al. 2008: 233-242.

Valvas lineales con extremos subcapitados, redondeados. VR: área axial lineal; área central transversalmente rectangular; rafe filiforme con las fisuras proximales levemente curvadas hacia la misma dirección. VSR: área axial angosta, lineal, área central transversalmente rectangular. Estrías radiales en ambas valvas, ligeramente más espaciadas en la zona media de la valva, formadas por 4 a 5 areolas elongadas, solo visibles al MEB.

Eje apical 13,1-14 $\mu \mathrm{m}$; eje transapical 4,1 $\mu \mathrm{m}$; estrías $28-33$ en $10 \mu \mathrm{m}$.

Stauroneis acuta Smith Fig. 2: S.

Patrick \& Reimer 1966: 367, Fig.31: 1; Van de Vijver et al. 2004: 17, Fig. 94: 1-3, Fig. 95: 1-4.

Valvas romboides con extremos angostos, redondeados. Pseudoseptos presentes. Área
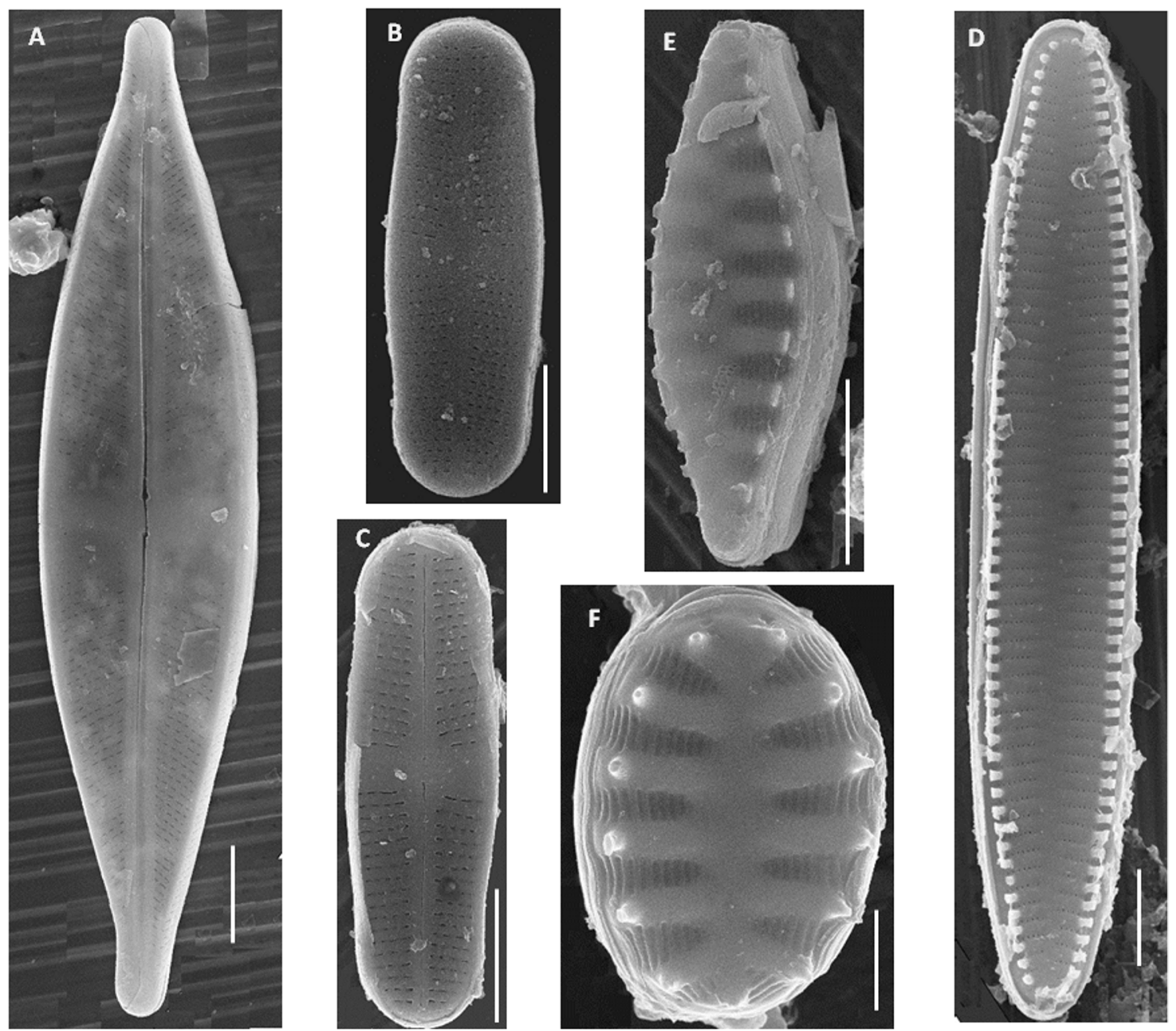

Fig. 4. A: Stauroneis aff. vandevijveri. B-C: Psammothidium abundans. D: Staurosira sp. 1. E: Staurosirella sp. 1. F: Staurosirella sp. 2. Escala $=5 \mu \mathrm{m}$; excepto $\mathrm{F}$ Escala $=2 \mu \mathrm{m}$. 
Bol. Soc. Argent. Bot. 50 (2) 2015

\begin{tabular}{|c|c|c|c|c|c|c|c|c|c|c|c|c|c|c|c|c|c|c|c|c|c|c|c|c|c|c|c|c|}
\hline స్ & & $\times$ & $\times$ & & $\times$ & $\times$ & $\times$ & & & $\times$ & $\times$ & & & $x$ & $x$ & & & & $\times$ & & & & $\times$ & $x$ & & & & \\
\hline 이 & & $\times$ & $x$ & & $x$ & $x$ & $x$ & & & $x$ & $x$ & & & & $x$ & & & & & & & & $\times$ & $\times$ & & & & \\
\hline : & & $\times$ & $\times$ & & & $\times$ & $x$ & & & & $x$ & & $\times$ & & $\times$ & $x$ & & $\times$ & & & & & $\times$ & $\times$ & & & $\times$ & \\
\hline N & & $x$ & $x$ & & $x$ & & & & & & $x$ & & & & $x$ & $x$ & & & & & & & $x$ & $x$ & & & $\times$ & \\
\hline$\stackrel{N}{ }$ & & $\times$ & $\times$ & $\times$ & & $\times$ & $x$ & & & & $\times$ & $x$ & & & $\times$ & & & & & & & & $\times$ & $\times$ & & & $\times$ & \\
\hline N & & $\times$ & $x$ & $\times$ & & $\times$ & & & & & $\times$ & & & & $x$ & & & & & & & $x$ & $\times$ & $\times$ & & & $x$ & \\
\hline 오 & & $x$ & $x$ & & $\times$ & $x$ & $\times$ & & & & $x$ & & & & $x$ & & & & & & & & $x$ & $\times$ & & & & \\
\hline$\stackrel{9}{-}$ & & $\times$ & $\times$ & & & $\times$ & $\times$ & & & & $x$ & & & & $x$ & & & & & $x$ & & & $\times$ & $\times$ & & & & \\
\hline$\stackrel{\infty}{\sim}$ & & $\times$ & $\times$ & $x$ & $\times$ & $\times$ & & & & & $x$ & $x$ & & & $x$ & $x$ & & & & $x$ & & $x$ & $x$ & $x$ & & & & \\
\hline$\mp$ & & $\times$ & $\times$ & & $x$ & $\times$ & $x$ & & & $\times$ & $\times$ & & & & $x$ & $\times$ & & & & & & & $\times$ & $\times$ & & & & \\
\hline$\stackrel{0}{\circ}$ & & $\times$ & $x$ & & $x$ & $\times$ & & & & $\times$ & $x$ & & & & $\times$ & $x$ & & & & & & & $x$ & $x$ & & & & \\
\hline$\stackrel{20}{2}$ & & $\times$ & $\times$ & & & $x$ & & & & $x$ & $x$ & & & & $x$ & & & & & & & & $x$ & $\times$ & & $x$ & & \\
\hline \pm & & $\times$ & $\times$ & & & $\times$ & & & & $x$ & $x$ & & & & $x$ & & & & & & & & $x$ & $\times$ & & & $x$ & \\
\hline$\stackrel{m}{=}$ & & $x$ & $\times$ & & $\times$ & $x$ & $\times$ & & & & $x$ & & & & $x$ & & & & & & & $x$ & $\times$ & $x$ & & & $\times$ & \\
\hline$\stackrel{\sim}{2}$ & & $\times$ & $x$ & & $x$ & $\times$ & $\times$ & & & & $x$ & & & $x$ & $\times$ & $\times$ & & $x$ & & & & & $x$ & $x$ & & & $x$ & \\
\hline$\mp$ & & $\times$ & $\times$ & & $x$ & $\times$ & $\times$ & & & & $x$ & & $x$ & & $x$ & $x$ & $x$ & & & & & & $\times$ & $\times$ & & & $\times$ & \\
\hline 우 & & $\times$ & $\times$ & & $x$ & $\times$ & $\times$ & & & & $x$ & & & & $x$ & $\times$ & & & & $\times$ & & & $x$ & $x$ & & & $\times$ & \\
\hline 0 & & $\times$ & $x$ & & $\times$ & $\times$ & & & & & $x$ & & $\times$ & & $x$ & & & & & & & & $\times$ & $\times$ & & & $x$ & \\
\hline$\infty$ & & $\times$ & $\times$ & & $\times$ & $\times$ & $\times$ & & & $\times$ & $\times$ & & $\times$ & & $\times$ & $\times$ & & & & & & & $\times$ & $\times$ & & $\times$ & $\times$ & \\
\hline$\wedge$ & $\times$ & $\times$ & $\times$ & & & $\times$ & $\times$ & & & $\times$ & $x$ & & & & $x$ & $x$ & & & & & & & $\times$ & $\times$ & & & $\times$ & \\
\hline 0 & $\times$ & $\times$ & $\times$ & & & $\times$ & & & $\times$ & $x$ & $x$ & & & & $\times$ & $x$ & & & & & & & $\times$ & $\times$ & $\times$ & & $\times$ & \\
\hline in & & $\times$ & $\times$ & & & $\times$ & $x$ & & & $\times$ & $x$ & & & & $\times$ & & & & & & & & $\times$ & $\times$ & & $x$ & & \\
\hline$\nabla$ & & $\times$ & $\times$ & & & $\times$ & & & & & $x$ & & & & $\times$ & $x$ & & & & $x$ & & & $\times$ & $\times$ & & $x$ & & \\
\hline m & & $x$ & $x$ & & $\times$ & & $\times$ & & & & $x$ & & & & $x$ & $x$ & & & $x$ & & $x$ & $x$ & $x$ & $x$ & & & $x$ & \\
\hline$N$ & & $\times$ & $\times$ & & $\times$ & $\times$ & $\times$ & $\times$ & & $x$ & $\times$ & & & & $\times$ & $x$ & & & & & & & $x$ & $x$ & $x$ & $x$ & & $\times$ \\
\hline- & & $\times$ & $x$ & & $x$ & $\times$ & $\times$ & & & $x$ & & & & & $x$ & $x$ & & & & & & & $x$ & $\times$ & & & & \\
\hline 0 & & $\times$ & $\times$ & & & $\times$ & $\times$ & & & $x$ & & & $x$ & & $x$ & $\times$ & & & & & & & $x$ & $\times$ & & & & \\
\hline 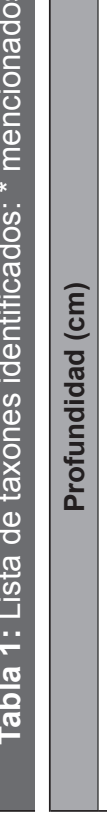 & 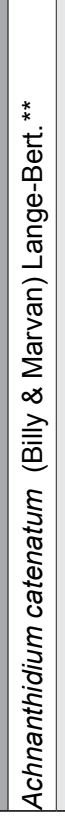 & 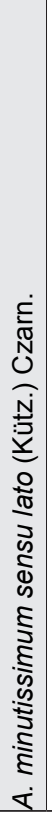 & 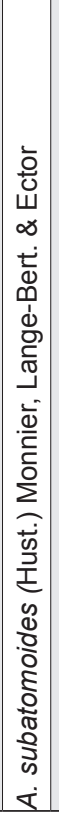 & 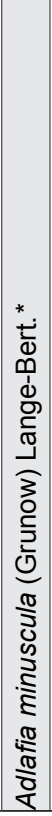 & 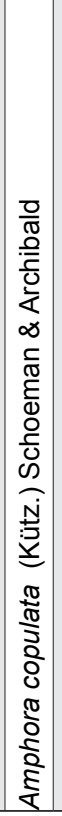 & 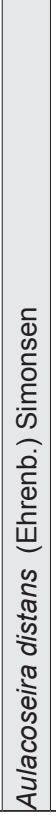 & 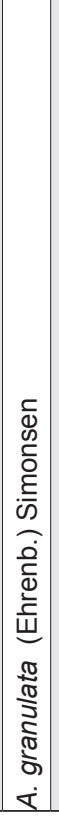 & 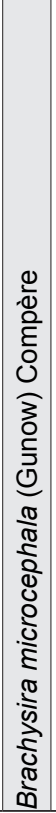 & 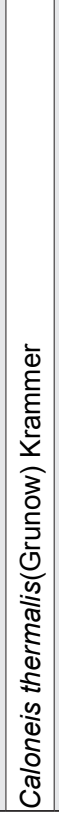 & 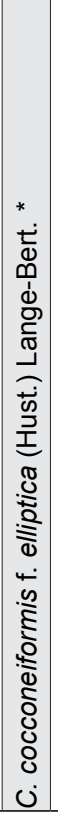 & 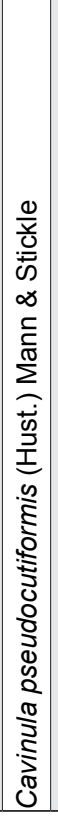 & 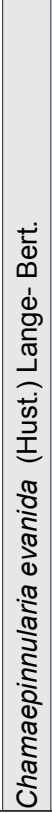 & 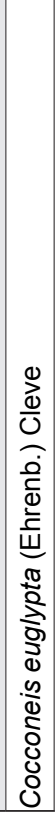 & 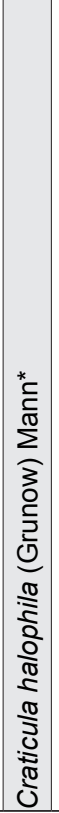 & 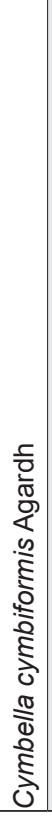 & 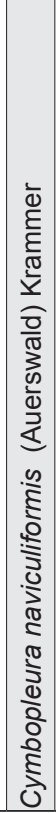 & 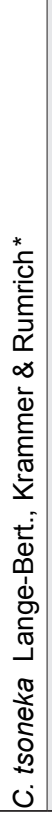 & 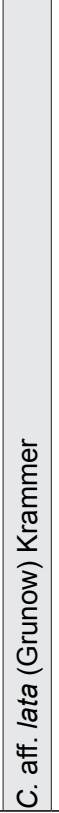 & 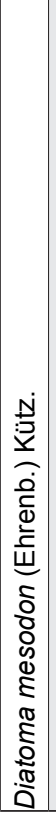 & 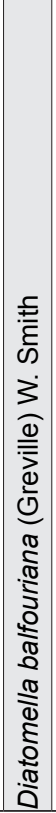 & 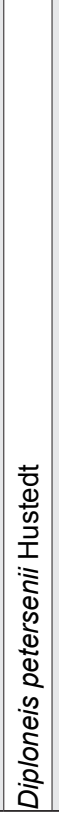 & 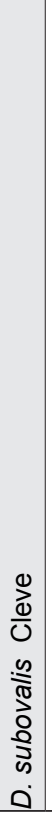 & 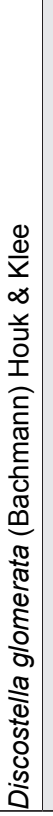 & 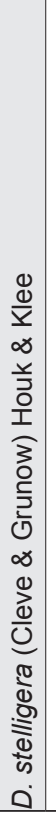 & 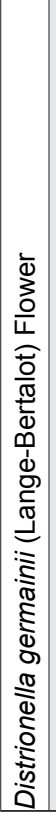 & 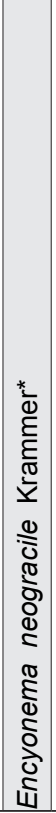 & 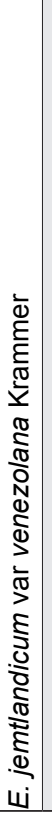 & 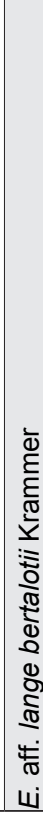 \\
\hline
\end{tabular}




\begin{tabular}{|c|c|c|c|c|c|c|c|c|c|c|c|c|c|c|c|c|c|c|c|c|c|c|c|c|c|c|}
\hline ల్ల & & & & & $\times$ & $x$ & & $x$ & & & & & & & & $\times$ & $x$ & & & & & & & & $x$ & \\
\hline 이 & $x$ & & & $\times$ & $\times$ & & & & & & & & & & & $x$ & & & & & & $x$ & & & & \\
\hline జ్ & $x$ & & & & $x$ & $x$ & & & $x$ & & & & & & & $x$ & & $x$ & & & & & & & & \\
\hline $\mathscr{N}$ & $x$ & & & & $x$ & $\times$ & $x$ & & & & & & $\times$ & & & $x$ & $\times$ & $\times$ & $x$ & & $\times$ & $\times$ & & & & \\
\hline N & $x$ & & & & $x$ & & & $x$ & & & $x$ & & & & & $x$ & $x$ & $x$ & & & & & & & & \\
\hline $\mathcal{N}$ & $x$ & & & & $x$ & $\times$ & & & & & & & & & $\times$ & $x$ & & & & & & $x$ & & & $x$ & \\
\hline ని & $x$ & & & & $x$ & $x$ & & & & & & & & & & $x$ & & & & & & & & & & \\
\hline$\stackrel{2}{\square}$ & & & & $\times$ & $x$ & & & & & & & & & & & $x$ & & & & & & & & & $\times$ & \\
\hline$\stackrel{\infty}{\leftarrow}$ & $x$ & & & $x$ & $x$ & & & & & & $x$ & & & & & $x$ & & & $x$ & & & $x$ & & & & \\
\hline$₹$ & $x$ & & & $\times$ & $\times$ & & & & & & & & $\times$ & & & $\times$ & & & & & & & & & & \\
\hline$\stackrel{\odot}{\leftarrow}$ & $x$ & & & & $x$ & & & & & & & & & & $x$ & $\times$ & & & & & & $\times$ & & & & \\
\hline$\stackrel{2}{2}$ & $x$ & & & $x$ & $x$ & & & $x$ & & & & & & & & $x$ & & & & & & $x$ & & & & \\
\hline 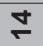 & $x$ & & & & $x$ & & & & & & & & & & & $x$ & & & & & & & & & & \\
\hline$\stackrel{m}{=}$ & $x$ & & & & $x$ & & & & & & & & & & & $\times$ & & & & & & $x$ & & & $x$ & $x$ \\
\hline 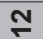 & $x$ & & & & $x$ & $x$ & & & & & & $x$ & & & & $x$ & $x$ & & & & & $x$ & & & & \\
\hline$F$ & $x$ & & & & $x$ & & & & & & & & $x$ & & & $x$ & & & & & & & $x$ & & & \\
\hline 우 & $x$ & & & & $x$ & & $x$ & & & & & & $x$ & & & $x$ & $x$ & & & & & $x$ & & & & \\
\hline o & $x$ & & & & $x$ & $x$ & & & & & & & & & & $x$ & & $x$ & & & & $x$ & & & $\times$ & $x$ \\
\hline$\infty$ & $x$ & & & $\times$ & $x$ & $x$ & & & & & & & $x$ & & & $x$ & & & & & & & & & & \\
\hline$\Lambda$ & $\times$ & $\times$ & & & $x$ & $\times$ & & & & & & & & $\times$ & & $x$ & $\times$ & & & & & & & $x$ & & \\
\hline 0 & $x$ & & & & $x$ & $x$ & & & & $\times$ & & & & & & $x$ & $x$ & $x$ & & & & & & $x$ & & \\
\hline in & $x$ & & & & $\times$ & $x$ & & & $\times$ & $x$ & & & & & & $x$ & & $x$ & & & & $\times$ & & & & $\times$ \\
\hline$\nabla$ & $x$ & & $x$ & & $x$ & $\times$ & & & & $x$ & & $\times$ & & & & $x$ & & & & $\times$ & & $\times$ & & & & $\times$ \\
\hline$m$ & $x$ & & & & $x$ & $\times$ & & & & & & & $\times$ & & & $x$ & $\times$ & $\times$ & & & $\times$ & $\times$ & & & $\times$ & \\
\hline$N$ & $x$ & & & & & $\times$ & $x$ & & & & & & & $\times$ & & $x$ & & & & & & $x$ & & & & $x$ \\
\hline$\sigma$ & $x$ & & & & $x$ & $x$ & & $x$ & & & & & & & & $x$ & $\times$ & $x$ & & & & $x$ & & & & \\
\hline 0 & & & & & & $x$ & & & & & & & & & & $\times$ & & $x$ & & & & $x$ & & & & \\
\hline 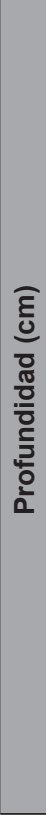 & 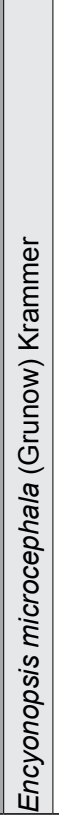 & 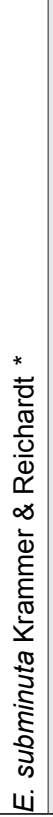 & 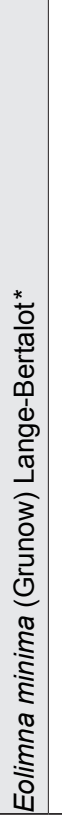 & 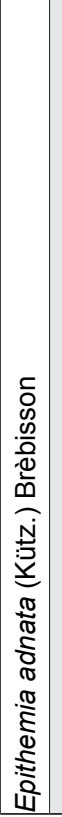 & 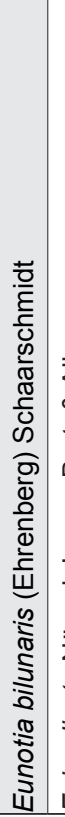 & 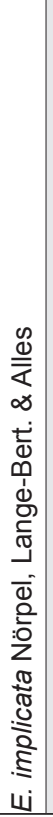 & 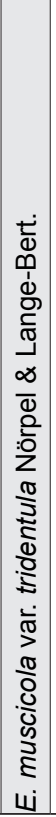 & 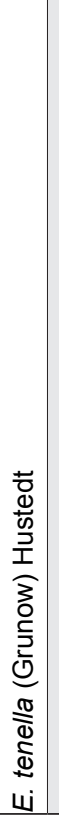 & 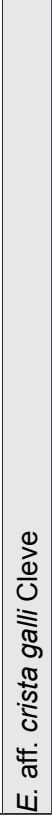 & 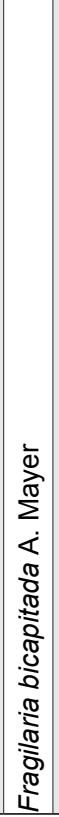 & 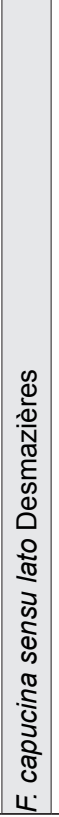 & 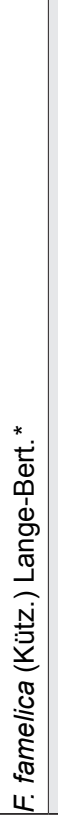 & 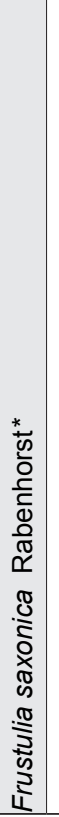 & 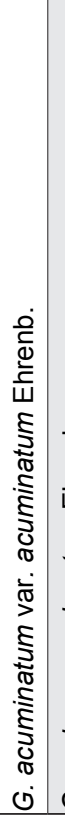 & 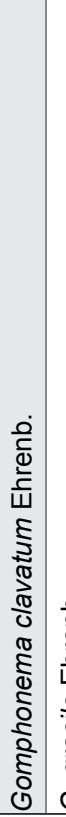 & 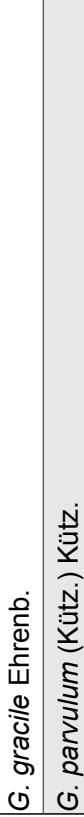 & 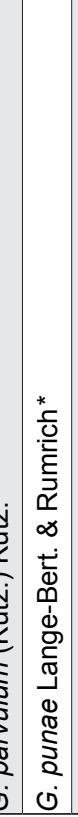 & 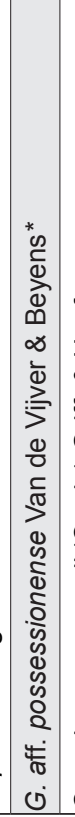 & 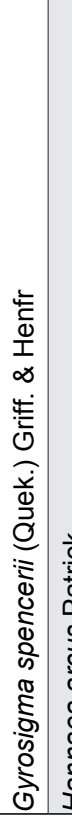 & 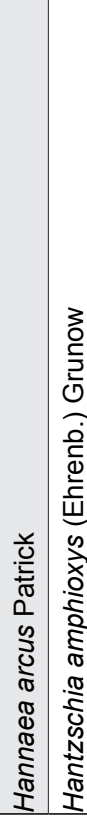 & 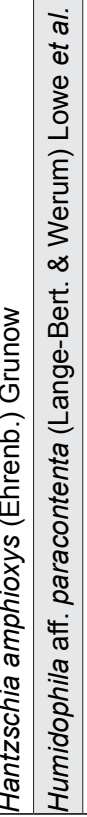 & 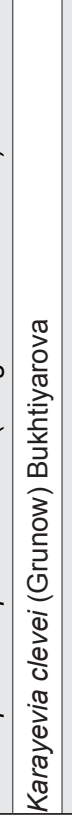 & 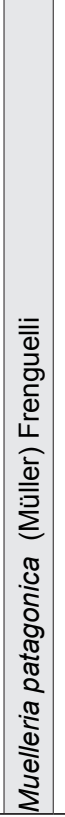 & 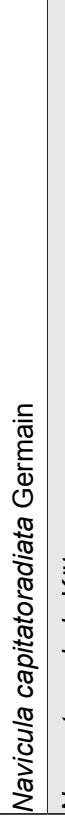 & 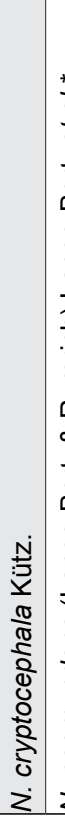 & 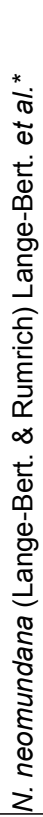 \\
\hline
\end{tabular}


Bol. Soc. Argent. Bot. 50 (2) 2015

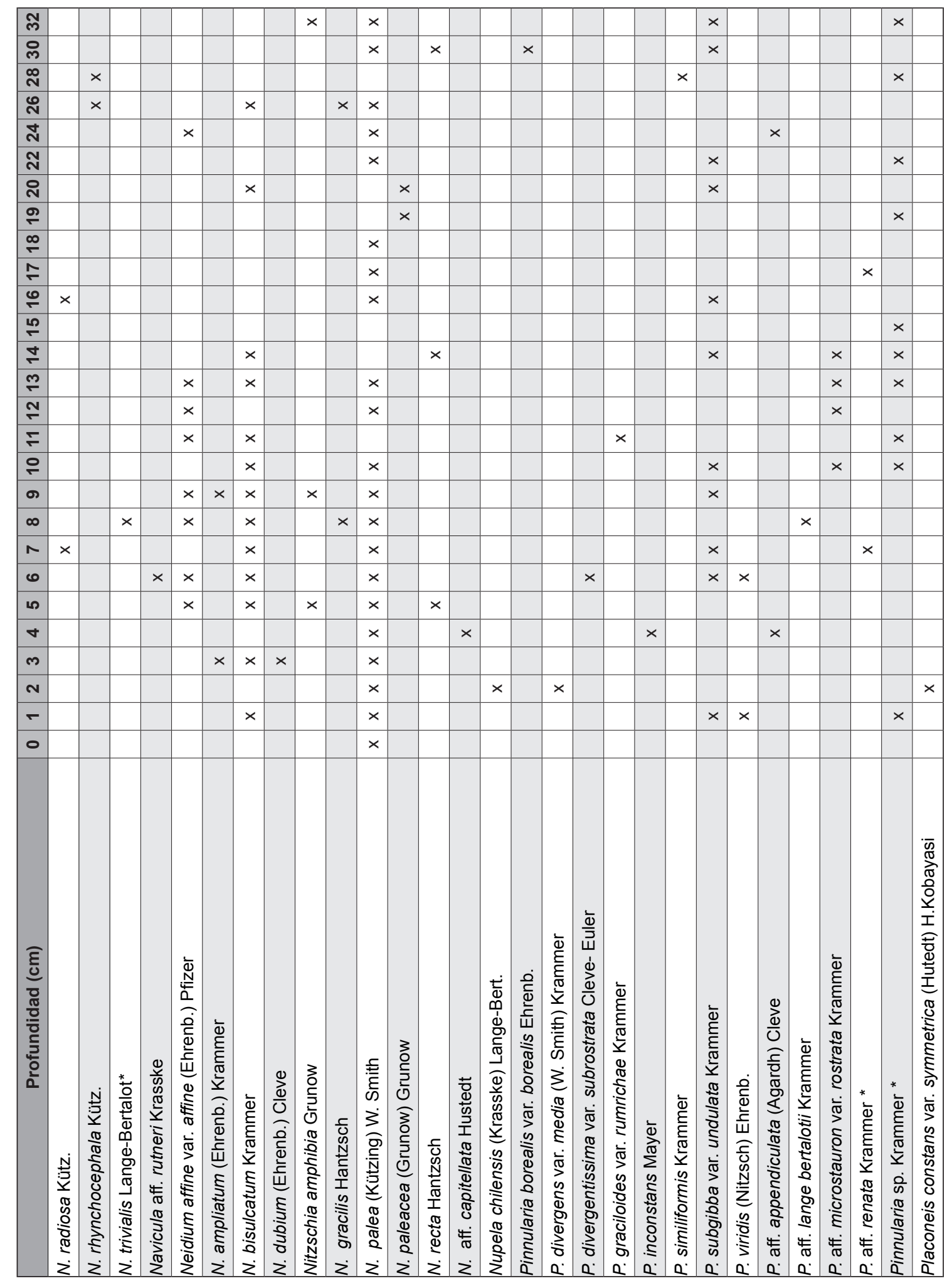




\begin{tabular}{|c|c|c|c|c|c|c|c|c|c|c|c|c|c|c|c|c|c|c|c|c|c|c|c|c|c|c|c|c|}
\hline స్ల & & $\times$ & & & & & & & & $\times$ & & & $\times$ & & & & & & & & & & & & & & & \\
\hline ి्ల & & $x$ & & & & & & & & $x$ & & & & $x$ & & & $\times$ & $\times$ & & & & & & & & & & $\times$ \\
\hline న & & $\times$ & & & & & & & & $\times$ & & & & & & $\times$ & & & & & & & & & & & & $x$ \\
\hline స & & $\times$ & & & & & & & & $x$ & & & & & $\times$ & & & & & & & & & & & & & $x$ \\
\hline$\stackrel{\sim}{N}$ & & $\times$ & & & & & & & $\times$ & $\times$ & & & & $x$ & & & & & & & & & $\times$ & & & & & $\times$ \\
\hline ส & & $\times$ & & & & & & & & $\times$ & $x$ & & $\times$ & & & $\times>$ & $\times$ & $x$ & & & & & & & & & & $\times$ \\
\hline กิ & & & & & & & & & & $\times$ & & & & & & & & & & & & & & & & & & $\times$ \\
\hline$\stackrel{2}{2}$ & & $\times$ & & & & & & & & $x$ & & & $\times$ & & & $\times$ & & & & & $\times$ & & & & & & & $x$ \\
\hline$\stackrel{\infty}{\sim}$ & & $\times$ & $\times$ & & & & & & & $\times$ & $\times$ & & & $x$ & & $\times \quad x$ & \begin{tabular}{c|c}
$\times$ & $\times$ \\
\end{tabular} & $\times \quad \times$ & & & & $\times$ & & & & & $\times$ & $\times$ \\
\hline$\approx$ & & & & $\times$ & & & & $\times$ & & $\times$ & & & $\times$ & & $x$ & & & & & & & & & & & & & $\times$ \\
\hline$\stackrel{\bullet}{\circ}$ & & & & & $\times$ & & & $x$ & & $\times$ & & & & & & $\times$ & & & & & & $x$ & & & & & & $x$ \\
\hline$\stackrel{2}{\stackrel{2}{2}}$ & & & $\times$ & & & & & & & $x$ & & & & & & & & & & & & & & $\times$ & & & & $x$ \\
\hline$\stackrel{+}{ \pm}$ & & & $\times$ & & & & & $x$ & & $\times$ & & & & & & & & $\times$ & & & & & & & & & & $x$ \\
\hline$\stackrel{m}{\stackrel{m}{2}}$ & & & & & & & & & & $x$ & & & $\times$ & $\times$ & & & & & & $\times$ & $\times$ & & & & & & & $x$ \\
\hline$\stackrel{\sim}{\simeq}$ & & $\times$ & & & & & & & & $x$ & $x$ & & $\times$ & & & $\times$ & & & & & & & & & & & & $\times$ \\
\hline$\mp$ & & & & & & & & & & $\times$ & & & & & & $\times$ & & & & & & & & & & & $\times$ & $\times$ \\
\hline 운 & $\times$ & & & $\times$ & & & & & & $x$ & & & $x$ & & $\times$ & & & & & & & & & & & & & $x$ \\
\hline a & & $\times$ & & & & & & & & $\times$ & & & $\times$ & & & & & & & & & & & & & & $\times$ & $x$ \\
\hline$\infty$ & & $\times$ & & & & & & & & $x$ & & $\times$ & & & & & & $\times$ & & & & & & & & & $\times$ & $\times$ \\
\hline$\Lambda$ & & & & & & & & & & $\times$ & & & & & $\times$ & & & & & $\times$ & & & & & & $\times$ & $\times$ & $\times$ \\
\hline 0 & & & $\times$ & & & $x$ & & & & $x$ & & & & & $\times$ & & & & & & & & & $x$ & & & $\times$ & \\
\hline n & & & & $\times$ & & & $x$ & & & $x$ & & & $\times$ & & & & & & & & & & & & & & $x$ & $\times$ \\
\hline$\nabla$ & & $\times$ & & $x$ & & & $\times$ & & & $\times$ & $\times$ & & & & & $\times$ & & & & & & & $\times$ & $x$ & & & $\times$ & $\times$ \\
\hline$m$ & & & $\times$ & $\times$ & $\times$ & & & & & $\times$ & & & & & $\times$ & & & & $\times$ & & & & & $\times$ & $\times$ & & $\times$ & $\times$ \\
\hline$N$ & & & & $\times$ & & & & & & $x$ & & & & & $x$ & & & & & $\times$ & & & & & & & $\times$ & $\times$ \\
\hline- & & & & $x$ & & & & & & $x$ & & & & $x$ & & & & & & & & & & & & & $\times$ & $\times$ \\
\hline 0 & & & $\times$ & & & & & & & $\times$ & & & & $\times$ & & & & & & & & & & & & & $\times$ & $\times$ \\
\hline 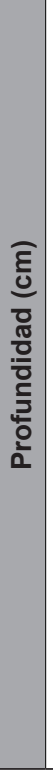 & 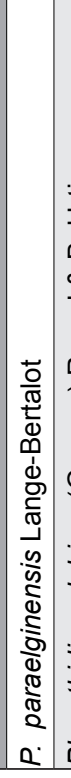 & 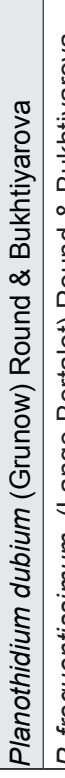 & 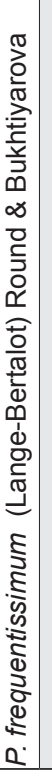 & 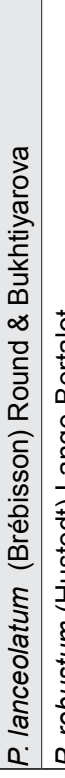 & 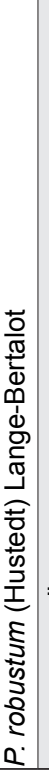 & 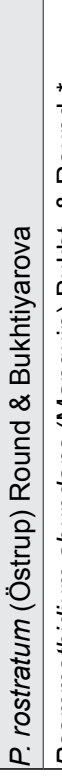 & 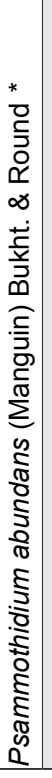 & 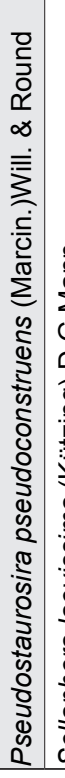 & 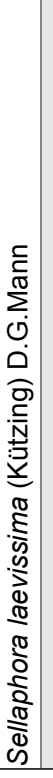 & 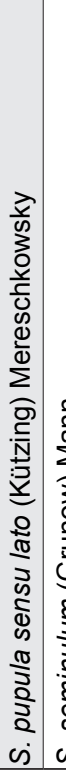 & 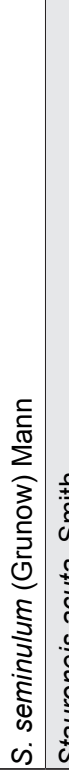 & 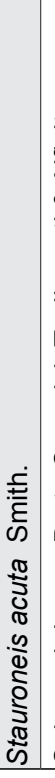 & 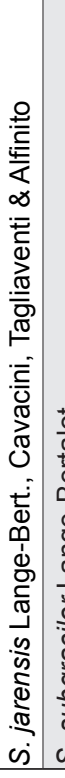 & 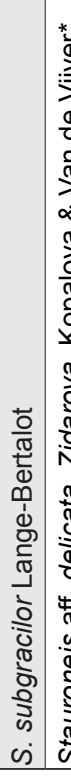 & 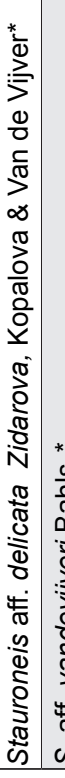 & 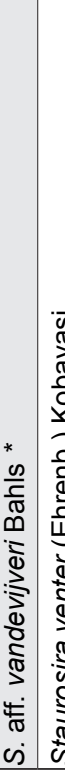 & 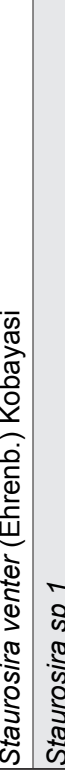 & 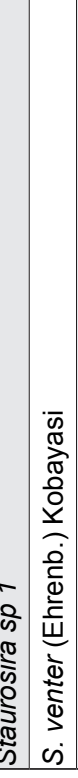 & 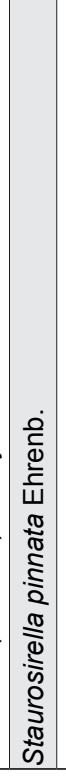 & 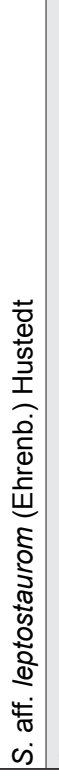 & 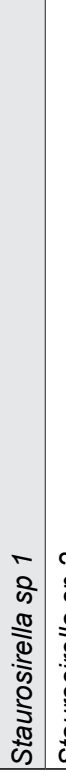 & 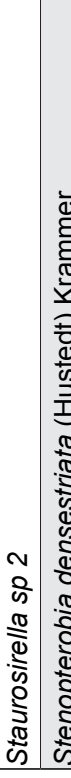 & 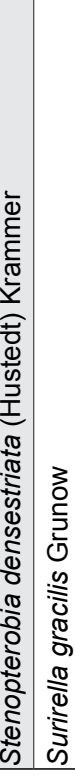 & 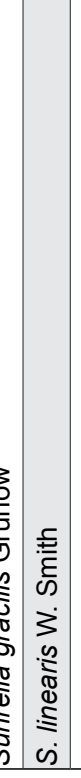 & 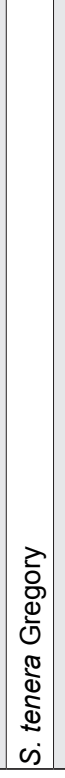 & 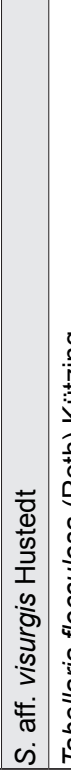 & 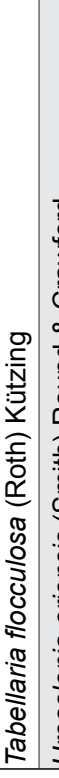 & 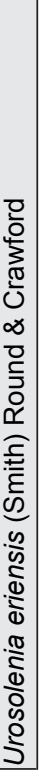 \\
\hline
\end{tabular}


Bol. Soc. Argent. Bot. 50 (2) 2015

\begin{tabular}{|c|c|c|c|c|c|c|c|c|c|c|c|c|c|c|c|}
\hline స్ల్ & 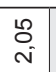 & & $\begin{array}{l}\overline{5} \\
0 \\
0\end{array}$ & in & $\begin{array}{l}\infty \\
\infty \\
0 \\
0 \\
N\end{array}$ & : & : & $\stackrel{\mathscr{R}}{\leftarrow}$ & $\begin{array}{l}\stackrel{D}{N} \\
\stackrel{N}{0}\end{array}$ & $\begin{array}{l}\text { m. } \\
\text { in }\end{array}$ & 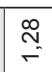 & $\begin{array}{l}\text { के } \\
\text { m. }\end{array}$ & $\begin{array}{l}8 \\
+ \\
+\end{array}$ & : & \\
\hline ల్లి & $\begin{array}{l}\text { fo } \\
\text { bi }\end{array}$ & & $\begin{array}{l}\text { 足 } \\
\text { s. }\end{array}$ & $\begin{array}{l}\Gamma \\
0 \\
0\end{array}$ & 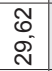 & $\underset{\mathcal{F}}{-}$ & $\begin{array}{l}8 \\
0\end{array}$ & 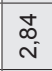 & $\begin{array}{l}8 \\
0\end{array}$ & $\begin{array}{l}\infty \\
\infty \\
\tilde{m}\end{array}$ & 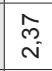 & $\begin{array}{l}N \\
\infty^{-}\end{array}$ & $\underset{\sim}{\stackrel{8}{-}}$ & 8 & s \\
\hline$\stackrel{\infty}{\sim}$ & $\begin{array}{c}\bar{v} \\
0 \\
0\end{array}$ & $\overline{1}$ & 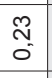 & $\begin{array}{l}8 \\
0 \\
0\end{array}$ & $\begin{array}{l}\text { बे } \\
\text { ले }\end{array}$ & 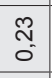 & $\begin{array}{l}8 \\
0\end{array}$ & $\underset{-}{-}$ & $\begin{array}{l}0 \\
0 \\
0 \\
0\end{array}$ & $\begin{array}{l}\text { O } \\
\text { Ñ }\end{array}$ & $\stackrel{\circ}{=}$ & $\begin{array}{l}\infty \\
\sim \\
\infty \\
\end{array}$ & $\begin{array}{l}\text { N } \\
\text { m }\end{array}$ & $\begin{array}{l}8 \\
0\end{array}$ & \\
\hline \& & 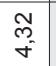 & : & $\stackrel{8}{8}$ & $\widetilde{\sigma}$ & $\begin{array}{l}\infty \\
\stackrel{\infty}{\infty} \\
\infty^{\infty}\end{array}$ & $\begin{array}{l}\infty \\
\stackrel{\infty}{+} \\
0\end{array}$ & 8 & 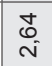 & $\begin{array}{l}\infty \\
\substack{+0}\end{array}$ & $\begin{array}{l}\stackrel{+}{\pi} \\
\tilde{N}\end{array}$ & $\stackrel{\circ}{i}$ & $\begin{array}{l}\infty \\
m \\
0\end{array}$ & $\begin{array}{l}\text { Do } \\
\text { in }\end{array}$ & 8 & \\
\hline$\stackrel{\text { I }}{2}$ & 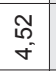 & \begin{tabular}{l}
$\swarrow 0$ \\
\hdashline \\
-
\end{tabular} & 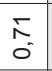 & $\hat{R}_{0}$ & $\begin{array}{l}\tilde{N} \\
\stackrel{N}{N}\end{array}$ & $\begin{array}{l}\stackrel{ \pm}{N} \\
\dot{0}\end{array}$ & 8 & $\begin{array}{l}\tilde{N} \\
\underset{i}{*}\end{array}$ & 8 & $\begin{array}{l}\text { Nे } \\
\text { Ni }\end{array}$ & 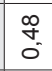 & \begin{tabular}{l}
$\infty$ \\
\multirow{0}{0}{} \\
$\stackrel{0}{0}$
\end{tabular} & $\begin{array}{c}m \\
m \\
m\end{array}$ & $\begin{array}{l}8 \\
0\end{array}$ & ᄂ \\
\hline N & 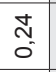 & $\begin{array}{l}\bar{\infty} \\
\aleph^{\infty}\end{array}$ & 8 & $\underset{\mathrm{N}}{\mathbf{D}}$ & $\begin{array}{l}m \\
m \\
m \\
m\end{array}$ & $\begin{array}{l}\hat{\sigma}_{-} \\
-\end{array}$ & 8 & $\stackrel{\infty}{\sim} \underset{\sim}{\sim}$ & 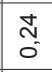 & 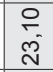 & $\stackrel{\vec{T}}{\stackrel{\Delta}{N}}$ & $\begin{array}{l}\hat{i} \\
\tilde{m} \\
\stackrel{n}{2}\end{array}$ & $\begin{array}{l}\tilde{c} \\
\text { in }\end{array}$ & 8 & 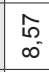 \\
\hline ని & 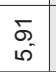 & ชู & $\begin{array}{l}\Sigma \\
0 \\
0\end{array}$ & $\begin{array}{l}\infty \\
\stackrel{\infty}{\infty}\end{array}$ & $\begin{array}{l}\stackrel{\infty}{\circ} \\
\stackrel{\rho}{\rho}\end{array}$ & $\underset{\sim}{\stackrel{v}{r}}$ & $\begin{array}{l}8 \\
0\end{array}$ & $\begin{array}{l}\text { ஜ̊ } \\
\stackrel{\circ}{\circ}\end{array}$ & $\begin{array}{l}\hat{f} \\
0\end{array}$ & $\begin{array}{l}\bar{\delta} \\
\bar{j}\end{array}$ & $\begin{array}{l}\stackrel{\leftrightarrow}{\Omega} \\
0 \\
0\end{array}$ & $\begin{array}{l}\stackrel{M}{\sim} \\
\stackrel{5}{\sim}\end{array}$ & \begin{tabular}{l}
$\tilde{N}$ \\
\multirow{\sigma}{*}{}
\end{tabular} & $\begin{array}{l}8 \\
0\end{array}$ & 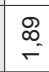 \\
\hline$\stackrel{2}{\circ}$ & $\begin{array}{l}\stackrel{2}{2} \\
b^{\circ}\end{array}$ & $\stackrel{\circ}{\circ}$ & $\underset{\sim}{\stackrel{ }{\sim}}$ & $\underset{\sim}{N}$ & 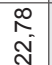 & \&. & \& & $\begin{array}{l}\stackrel{8}{0} \\
\stackrel{\circ}{\circ}\end{array}$ & : & $\begin{array}{l}0 \\
\text { م⿱ } \\
\text { on }\end{array}$ & $\underset{f}{f}$ & $\frac{\infty}{\sigma}$ & $\stackrel{N}{\stackrel{N}{m}}$ & $\begin{array}{l}8 \\
0\end{array}$ & $\begin{array}{l}\text { Dे } \\
\text { in }\end{array}$ \\
\hline$\stackrel{\infty}{\circ}$ & 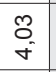 & $\underset{\text { Ta }}{=}$ & 8 & $\begin{array}{l}\text { fo } \\
0\end{array}$ & $\begin{array}{l}0 \\
\infty \\
\omega^{2}\end{array}$ & $\stackrel{\infty}{\leftarrow}$ & 8 & $\begin{array}{l}\overline{1} \\
0\end{array}$ & $\begin{array}{l}8 \\
0\end{array}$ & $\begin{array}{l}\bar{\infty} \\
\bar{m} \\
\end{array}$ & $\begin{array}{l}8 \\
0\end{array}$ & $\begin{array}{l}\mathscr{8} \\
\text { N }\end{array}$ & $\begin{array}{l}\overline{6} \\
i\end{array}$ & $\begin{array}{l}\text { f } \\
0\end{array}$ & $\stackrel{8}{\circ}$ \\
\hline$\mp$ & $\begin{array}{l}\Gamma \\
\hat{0}\end{array}$ & d & $\begin{array}{l}\mathscr{8} \\
0 \\
0\end{array}$ & $\begin{array}{l}N \\
\tilde{O}\end{array}$ & 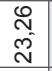 & 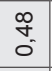 & 8 & 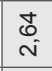 & 8 & $\begin{array}{l}\mathbb{N} \\
\mathbb{N}\end{array}$ & $\underset{m}{\stackrel{N}{*}}$ & $\begin{array}{l}0 \\
\hat{0} \\
0 \\
0\end{array}$ & $\begin{array}{l}\infty \\
\stackrel{+}{+} \\
\end{array}$ & 8 & 恋 \\
\hline$\div$ & $\underset{\sim}{J}$ & $\begin{array}{l}\text { f } \\
\text { m. }\end{array}$ & 8 & 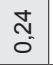 & $\begin{array}{l}\text { Oo } \\
\stackrel{N}{N}\end{array}$ & $\begin{array}{l}\infty \\
\stackrel{\infty}{0} \\
0\end{array}$ & 8 & 亲 & 8 & $\begin{array}{l}\text { ô } \\
\text { o }\end{array}$ & $\stackrel{\underset{N}{\sim}}{\sim}$ & \begin{tabular}{l}
$\stackrel{m}{+}$ \\
\multirow{\leftarrow}{\leftarrow}{}
\end{tabular} & $\stackrel{m}{S}$ & 8 & $\stackrel{\infty}{\infty}$ \\
\hline$\stackrel{2}{2}$ & $\frac{m}{50}$ & $\stackrel{\stackrel{\mathscr{N}}{\mathrm{N}}}{\mathrm{F}}$ & $\begin{array}{l}8 \\
0\end{array}$ & 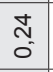 & $\begin{array}{l}\text { \& } \\
\stackrel{N}{0}\end{array}$ & $\underset{\sim}{\mathbb{N}}$ & 8 & $\underset{\leftarrow}{\stackrel{f}{-}}$ & $\begin{array}{l}8 \\
0\end{array}$ & $\begin{array}{l}\text { \& } \\
\text { \&े }\end{array}$ & $\stackrel{\stackrel{\leftrightarrow}{\circ}}{\sim}$ & $\begin{array}{l}8 \\
=\end{array}$ & i & 8 & $\begin{array}{l}\hat{0} \\
\dot{m}\end{array}$ \\
\hline$\stackrel{\nabla}{ }$ & $\underset{\sim}{\stackrel{\rho}{m}}$ & $\underset{\sim}{\sim}$ & $\begin{array}{l}8 \\
0 \\
0\end{array}$ & $\begin{array}{l}\stackrel{\mathfrak{L}}{2} \\
0\end{array}$ & $\begin{array}{l}\stackrel{J}{*} \\
\text { d }\end{array}$ & $\underset{-}{\stackrel{\circ}{-}}$ & O & $\stackrel{\circ}{\circ}$ & $\begin{array}{l}8 \\
0\end{array}$ & $\begin{array}{l}8 \\
\dot{e}\end{array}$ & $\begin{array}{l}\stackrel{2}{2} \\
0\end{array}$ & $\begin{array}{l}0 \\
0 \\
6 \\
5\end{array}$ & $\stackrel{g}{i}$ & $\begin{array}{l}8 \\
0\end{array}$ & $\stackrel{\infty}{\square}$ \\
\hline$\stackrel{m}{-}$ & 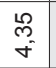 & 色 & $\stackrel{8}{\circ}$ & $\begin{array}{l}0 \\
0 \\
0\end{array}$ & $\begin{array}{l}\text { N } \\
\text { Ni }\end{array}$ & $\begin{array}{l}\mathbb{\infty} \\
\stackrel{\infty}{\leftarrow}\end{array}$ & $\begin{array}{l}8 \\
0\end{array}$ & $\begin{array}{l}0 \\
0 \\
0\end{array}$ & 8 & \begin{tabular}{|l|}
0 \\
0 \\
0
\end{tabular} & $\stackrel{m}{m}$ & $\begin{array}{l}\hat{\sigma} \\
\stackrel{F}{F}\end{array}$ & 胥 & $\begin{array}{l}8 \\
0 \\
0\end{array}$ & 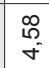 \\
\hline$\cong$ & $\begin{array}{c}\overline{+} \\
\sigma\end{array}$ & $\stackrel{\widehat{g}}{r}$ & 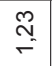 & $\frac{\pi}{\hat{O}}$ & $\begin{array}{l}\hat{0} \\
\infty^{-}\end{array}$ & $\begin{array}{l}\infty \\
\stackrel{\infty}{0} \\
0\end{array}$ & 8 & $\stackrel{N}{\stackrel{N}{\sim}}$ & $\begin{array}{l}\stackrel{n}{N} \\
0\end{array}$ & $\frac{\text { fo }}{5}$ & f́ & $\begin{array}{l}\text { 8 } \\
\text { o }\end{array}$ & $\begin{array}{l}8 \\
0 \\
\text { m. }\end{array}$ & $\begin{array}{l}8 \\
0\end{array}$ & $\begin{array}{l}\text { D. } \\
\text { in }\end{array}$ \\
\hline$=$ & $\begin{array}{l}\hat{0} \\
\infty^{\circ}\end{array}$ & 8 & $\begin{array}{l}\text { M } \\
\stackrel{0}{0}\end{array}$ & $\stackrel{5}{-}$ & $\frac{\vec{N}}{\stackrel{N}{N}}$ & $\underset{\sim}{\stackrel{N}{\sim}}$ & 8 & $\begin{array}{l}\infty \\
0 \\
0 \\
0\end{array}$ & $\begin{array}{l}8 \\
0\end{array}$ & $\frac{\vec{N}}{\stackrel{N}{N}}$ & $\Sigma$ & $\begin{array}{l}5 \\
5 \\
0\end{array}$ & $\begin{array}{l}9 \\
\stackrel{9}{*} \\
\forall\end{array}$ & $\begin{array}{l}\infty \\
0 \\
0 \\
0\end{array}$ & 丹 \\
\hline 우 & $\begin{array}{l}m \\
\stackrel{5}{\circ}\end{array}$ & 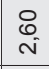 & $\Sigma_{0}$ & f́ & $\begin{array}{l}\text { bo } \\
\text { in }\end{array}$ & $\begin{array}{l}\stackrel{ \pm}{N} \\
\text { O }\end{array}$ & 8 & $\begin{array}{l}\text { L } \\
0 \\
0\end{array}$ & 8 & $\begin{array}{l}0 \\
\text { N } \\
\text { - }\end{array}$ & $\underset{\sim}{\stackrel{Y}{\sim}}$ & $\begin{array}{l}\text { I } \\
\mathcal{Z}\end{array}$ & \begin{tabular}{l}
$\tilde{O}$ \\
\multirow{\sigma}{*}{}
\end{tabular} & $\begin{array}{l}8 \\
0\end{array}$ & ָิ \\
\hline$\sigma$ & $\begin{array}{l}\frac{0}{F} \\
F\end{array}$ & $\stackrel{0}{\check{2}}$ & $\begin{array}{l}8 \\
0\end{array}$ & $\begin{array}{l}\bar{N} \\
0\end{array}$ & $\begin{array}{l}\text { q } \\
F\end{array}$ & $\stackrel{\leftrightarrow}{\circ}$ & $\begin{array}{l}8 \\
0\end{array}$ & $\begin{array}{l}\mathscr{2} \\
0 \\
0\end{array}$ & $\begin{array}{l}\infty \\
\text { o } \\
0\end{array}$ & N & $\begin{array}{l}\infty \\
\stackrel{\infty}{0}\end{array}$ & $\frac{\pi}{i}$ & $\begin{array}{l}0 \\
9 \\
i \\
5\end{array}$ & $\stackrel{9}{=}$ & ర్ \\
\hline$\infty$ & 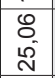 & बे & 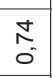 & $\begin{array}{l}\text { N } \\
0\end{array}$ & $\begin{array}{l}\stackrel{m}{y} \\
\infty \\
\stackrel{\infty}{c}\end{array}$ & $\stackrel{\mathbb{N}}{\stackrel{\sim}{\sim}}$ & 8 & f́ & 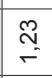 & $\begin{array}{l}\infty \\
\infty \\
\text { N }\end{array}$ & $\begin{array}{l}\text { N } \\
0\end{array}$ & $\begin{array}{l}\hat{L} \\
\text { of } \\
\end{array}$ & $\stackrel{N}{\stackrel{N}{\sim}}$ & $\underset{\mathbb{N}}{=}$ & $\begin{array}{l}\text { 乃 } \\
\text { m. }\end{array}$ \\
\hline$\Lambda$ & $\begin{array}{l}0 \\
\stackrel{0}{\tau}\end{array}$ & 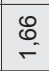 & 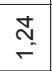 & $\stackrel{\text { fo }}{\leftarrow}$ & 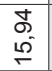 & $\stackrel{\circ}{\stackrel{\circ}{\leftarrow}}$ & 8 & $\begin{array}{c}\bar{m} \\
\text { m. }\end{array}$ & $\begin{array}{l}\infty \\
\infty \\
0 \\
0\end{array}$ & $\begin{array}{l}\text { N } \\
\text { in }\end{array}$ & $\stackrel{\underset{\sim}{\sim}}{\sim}$ & 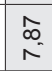 & 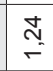 & 灾 & $\stackrel{\stackrel{t}{g}}{\sim}$ \\
\hline 0 & $\begin{array}{l}\overline{5} \\
0 \\
0\end{array}$ & 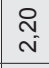 & $\begin{array}{l}8 \\
0\end{array}$ & $\begin{array}{l}\stackrel{N}{N} \\
\text { N }\end{array}$ & $\begin{array}{l}\bar{\sigma} \\
\stackrel{5}{\circ}\end{array}$ & $\begin{array}{l}M \\
0^{\prime}\end{array}$ & $\begin{array}{l}8 \\
0\end{array}$ & $\stackrel{\leftrightarrow}{\stackrel{\rho}{\circ}}$ & $\begin{array}{l}\Omega \\
\hat{0}\end{array}$ & $\begin{array}{c}\bar{\gamma} \\
\bar{\sigma}\end{array}$ & 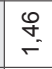 & $\begin{array}{l}\underset{\kappa}{F} \\
F\end{array}$ & \begin{tabular}{l}
0 \\
\hdashline \\
-
\end{tabular} & 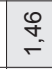 & : \\
\hline n & $\underset{f}{\stackrel{g}{+}}$ & & $\begin{array}{l}0 \\
0 \\
0 \\
0\end{array}$ & $\stackrel{\mathscr{L}}{\stackrel{2}{r}}$ & $\begin{array}{l}\text { f } \\
\stackrel{5}{F}\end{array}$ & 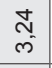 & 8 & $\begin{array}{l}0 \\
0 \\
0\end{array}$ & $\begin{array}{l}0 \\
0 \\
0\end{array}$ & $\begin{array}{l}\mathscr{8} \\
\infty \\
\text { యా }\end{array}$ & $\stackrel{\circ}{\circ}$ & $\begin{array}{l}\stackrel{2}{1} \\
\infty \\
\infty\end{array}$ & $\stackrel{\stackrel{2}{\sim}}{=}$ & $\begin{array}{l}\stackrel{2}{2} \\
0\end{array}$ & $\begin{array}{l}\stackrel{2}{2} \\
0\end{array}$ \\
\hline$\nabla$ & $\begin{array}{l}\Theta \\
\stackrel{m}{0}\end{array}$ & $\begin{array}{l}\Omega \\
0_{0}^{\circ}\end{array}$ & 8 & 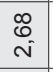 & $\begin{array}{l}8 \\
0 \\
\circ\end{array}$ & $\begin{array}{l}\Sigma \\
\digamma\end{array}$ & $\stackrel{20}{\circ}$ & $\underset{\sim}{\sim}$ & 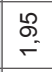 & $\begin{array}{l} \\
m \\
m \\
\tilde{m}\end{array}$ & 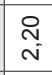 & $\begin{array}{l}\stackrel{\infty}{\infty} \\
\stackrel{\infty}{\infty}\end{array}$ & 㐫 & $\underset{i 5}{\stackrel{N}{5}}$ & 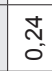 \\
\hline$m$ & 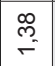 & : & $\begin{array}{l}m_{0} \\
m \\
0\end{array}$ & $\begin{array}{l}\stackrel{p}{\infty} \\
\text { m }\end{array}$ & $\begin{array}{l}8 \\
0 \\
0\end{array}$ & $\begin{array}{l}0 \\
\infty \\
0\end{array}$ & 8 & $\underset{\sim}{t}$ & î. & \begin{tabular}{l}
$\hat{\infty}$ \\
\multirow{N}{*}{}
\end{tabular} & $\begin{array}{l}\stackrel{8}{0} \\
\stackrel{6}{N}\end{array}$ & \begin{tabular}{l}
$\infty$ \\
0 \\
\multirow{0}{*}{}
\end{tabular} & $\begin{array}{l}0 \\
10 \\
0 \\
0\end{array}$ & $\begin{array}{l}\text { m } \\
\text { o }\end{array}$ & $\hat{0}$ \\
\hline$N$ & $\begin{array}{l}\text { m } \\
\text { in }\end{array}$ & $\underset{\sim}{\mathbb{N}}$ & $\stackrel{尺}{\Sigma}$ & $\underset{\Sigma}{\Sigma}$ & $\begin{array}{l}8 \\
\dot{N}\end{array}$ & $\begin{array}{l}\stackrel{\text { S }}{\text { m. }} \\
\end{array}$ & $\begin{array}{l}8 \\
0\end{array}$ & $\begin{array}{l}8 \\
0\end{array}$ & $\begin{array}{c}\infty \\
\substack{\infty\\
}\end{array}$ & 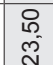 & $\begin{array}{l}\text { S } \\
\text { m) }\end{array}$ & $\begin{array}{l}0 \\
\stackrel{0}{\sigma}\end{array}$ & \begin{tabular}{l}
$\infty$ \\
$\infty$ \\
\multirow{\gamma}{*}{}
\end{tabular} & 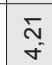 & $\hat{\theta}_{0}^{\circ}$ \\
\hline- & 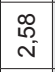 & 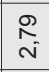 & $\underset{\substack{\infty \\
i}}{ }$ & 8 & $\underset{\delta}{\delta}$ & $\stackrel{N}{\stackrel{N}{\digamma}}$ & 8 & $\frac{10}{i}$ & 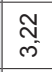 & $\begin{array}{l}\mathrm{N} \\
\mathrm{m}\end{array}$ & 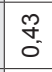 & $\begin{array}{l}\hat{\infty} \\
\omega^{-}\end{array}$ & 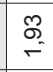 & 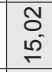 & $\underset{+}{\mathbb{N}}$ \\
\hline 0 & $\begin{array}{l}\hat{0} \\
\dot{m}\end{array}$ & $\begin{array}{l}\text { के } \\
\text { n. }\end{array}$ & $\underset{+}{\mathbb{N}}$ & 8 & $\begin{array}{l}0 \\
0 \\
0 \\
0\end{array}$ & $\begin{array}{l}8 \\
0 \\
0\end{array}$ & 8 & 8 & \begin{tabular}{l}
$\stackrel{2}{+}$ \\
\multirow{+}{*}{}
\end{tabular} & $\begin{array}{l}\text { 怘 } \\
\text { ల్ల }\end{array}$ & $\begin{array}{l}\text { 令 } \\
0\end{array}$ & $\begin{array}{l}\tilde{m} \\
0 \\
0\end{array}$ & $\begin{array}{l}\vec{d} \\
\text { m. }\end{array}$ & \begin{tabular}{l}
8 \\
\multirow{5}{*}{}
\end{tabular} & 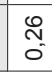 \\
\hline 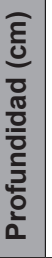 & 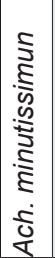 & 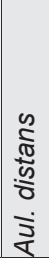 & 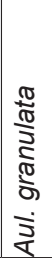 & 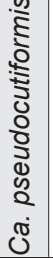 & $\begin{array}{l}\frac{2}{2} \\
0 \\
\mathbb{0} \\
\frac{\Phi}{w} \\
\tilde{D} \\
0 \\
0\end{array}$ & $\begin{array}{l}\frac{\pi}{0} \\
\frac{0}{0} \\
\frac{0}{0} \\
\frac{0}{0} \\
\text { ह } \\
\text { dे } \\
\bar{\omega}\end{array}$ & 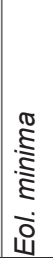 & 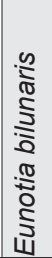 & 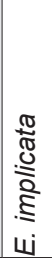 & 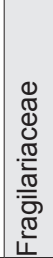 & 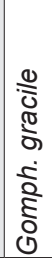 & 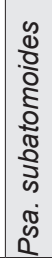 & $\begin{array}{l}\frac{\pi}{3} \\
\frac{2}{3} \\
\frac{0}{\Phi} \\
\omega \\
\omega\end{array}$ & 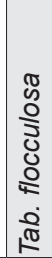 & 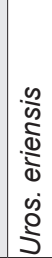 \\
\hline
\end{tabular}




\section{L. García y N. I. Maidana - Diatomeas subfósiles del Brazo Blest, Argentina}

axial lineal-lanceolada; estauro deltoide. Rafe ligeramente lateral. Estrías radiales en el centro de la valva, tornándose subparalelas cerca de los ápices.

Eje apical 159,5-160,6 $\mu \mathrm{m}$; eje transapical 21,3$23,5 \mu \mathrm{m}$; estrías $12-13$ en $10 \mu \mathrm{m}$; areolas 14 en $10 \mu \mathrm{m}$.

Stauroneis aff. delicata Zidarova, Kopalova \& Van de Vijver. Fig. 2: Q.

Zidarova et al. 2014, 191-207: Fig.77-92.

Valvas lanceoladas, con ápices rostrados. Rafe lateral. Área axial lineal; estauro rectangular. Estrías delicadas, radiales en toda la valva. Areolas transversalmente elongadas, poco distinguibles al MO.

Eje apical 41,3-51,1 $\mu \mathrm{m}$; eje transapical 8,5-9,1 $\mu \mathrm{m}$; estrías 23-24 en $10 \mu \mathrm{m}$; areolas 26 en $10 \mu \mathrm{m}$.

Obs.: Los ejemplares analizados se asemejan a $S$. delicata, descripta recientemente para la región Antártica. Sin embargo, nuestros ejemplares son ligeramente más anchos y se encontrarían en el extremo inferior de su rango de variación en cuanto a la densidad de estrías.

Stauroneis aff. vandevijveri Bahls. Fig. 2: R; Fig. 4: A.

Bahls 2011: http://westerndiatoms.colorado.edu; Van de Vijver et al. 2004: 21, Fig. 39: 1-10 (como Stauroneis "artic-anceps").

Valvas lanceoladas con extremos angostamente rostrados, sin pseudoseptos. Área axial estrecha, lineal; el área central forma un área rectangular (estauro), ligeramente expandida hacia los márgenes de la valva. Las ramas del rafe son filiformes. Los extremos proximales del rafe son rectos, los extremos terminales están curvados hacia el mismo lado. Estrías radiales en toda la valva. Las areolas, solo visibles al MEB, son elongadas transversalmente y de longitud irregular.

Eje apical 42,1-44,3 $\mu \mathrm{m}$; eje transapical 8,1-8,9 $\mu \mathrm{m}$; estrías 24-28 en $10 \mu \mathrm{m}$; areolas 20-28 en $10 \mu \mathrm{m}$

Obs.: El material observado se asemeja a $S$. vandevijverii en la forma general de la valva y los extremos y las dimensiones pero se diferencia por la mayor densidad de estrías y por la forma y menor densidad de las areolas.

\section{Staurosira sp. 1. Fig. 4: D.}

Valva lineal, con extremos cuneado-redondeados. Esternón angosto y lineal. Estrías paralelas en casi toda la valva, volviéndose subparalelas hacia los ápices. Las estrías son alternas a ambos lados del esternón y están formadas por areolas pequeñas, redondeadas. Espinas espatuladas presentes en cada interestría.

Eje apical 42,2 $\mu \mathrm{m}$; eje transapical $6,2 \mu \mathrm{m}$; estrías 14 en $10 \mu \mathrm{m}$.

Obs.: El ejemplar observado no se asemeja a ninguno de los descriptos en la bibliografía consultada. Es necesaria la observación de más material para poder identificar correctamente a este taxón.

Staurosirella sp. 1. Fig. 4: E.

Valva rómbico-lanceolada, con extremos subrostrados. Esternón casi imperceptible. Estrías paralelas y alternas respecto del esternón, formadas por areolas lineoladas. Espinas marginales planas ubicadas sobre las interestrías. Campos porosos apicales reducidos.

Eje apical 10,8 $\mu \mathrm{m}$; eje transapical $3,1 \mu \mathrm{m}$; estrías 10 en $10 \mu \mathrm{m}$.

Obs.: No hemos hallado en la bibliografía consultada taxones que se asemejen al ejemplar observado.

Staurosirella sp. 2. Fig. 4: F.

Valvas elípticas, con extremos anchamente redondeados. Esternón prominente. Estrías dispuestas en forma opuesta respecto del esternón, ligeramente radiales y compuestas por areolas lineoladas. Espinas cónicas presentes en cada interestría, en la zona de unión del manto con la valva.

Eje apical: 5,1-5,3 $\mu \mathrm{m}$; eje transapical 2,8-3,5 $\mu \mathrm{m}$; estrías 13-14 en $10 \mu \mathrm{m}$.

Obs.: El ejemplar observado no se asemeja a ninguno de los descriptos en la bibliografía consultada, por lo que es imprescindible la observación de más ejemplares para poder identificar correctamente a este taxón.

\section{Discusión y Conclusiones}

La taxonomía de las diatomeas es compleja $\mathrm{y}$, a pesar de todos los intentos realizados en las últimas décadas por mejorarla (Mann, 1999; Kociolek \& Spaulding, 2000; Vanormelingen et al., 2008), la delimitación de los taxones sigue 
haciéndose casi exclusivamente a partir de la observación de características morfológicas del frústulo, sin la compresión completa de los patrones de variabilidad morfológica que pueden presentar.

Achnanthidium catenatum, especie que mencionamos por primera vez para Argentina, era conocida en Europa, Asia y EEUU mientras que en Sudamérica, solo había sido citada para Colombia (Montoya-Moreno et al., 2013). Es una de las pocas especies planctónicas que forman cadenas, formadas por células unidas por su cara valvar. Esta característica, junto con su peculiar vista cingular, son los caracteres principales para su identificación. A. catenatum está considerada en Europa, como una especie invasora (Coste \& Ector 2000; Straub, 2002) pero, afortunadamente, no fue muy abundante en el testigo analizado $(<3 \%)$ y solo se la halló en 2 muestras subsuperficiales.

De acuerdo con los catálogos de Luchini \& Verona (1972), Tell (1985) y Vouilloud (2003), se han citado hasta el momento 560 especies de diatomeas para la provincia de Río Negro y 363 para la provincia de Neuquén, mientras que hay 111 registros de taxones comunes a ambas provincias.

Navicula neomundana, fue hallada por primera vez en la provincia de Santa Cruz (Echazú, 2012) por lo que este es el segundo registro de la especie en el país. Por otra parte, Fragilaria famelica, Navicula trivialis y Psamothidium abundans, fueron reportadas por Echazú (op. cit.) por primera vez para la Patagonia y en este trabajo ampliamos su distribución en la región.

El hallazgo de 18 nuevos registros para la Patagonia Norte pone en evidencia la necesidad de intensificar aún más los estudios taxonómicos, morfológicos y ecológicos de la biodiversidad diatomológica de la región, lo cual es objetivo de nuestros próximos trabajos en la Patagonia.

Dado que varios autores destacan la importancia de las diatomeas como indicadores biológicos (Spaulding \& Mc Knight, 1999; Smol \& Cumming, 2000), el conocimiento de su taxonomía será clave para integrarla con su distribución geográfica y características ecológicas. Particularmente, se debería considerar hacer un estudio más detallado de las Fragilariaceae, que muy probablemente tenga como consecuencia la descripción de nuevos taxones para la ciencia.

\section{Agradecimientos}

Este trabajo fue realizado en el marco del Proyecto CONICET-PIP 112-200801-02345. Agradecemos a su directora, Dra. Julieta Massaferro, por proveernos las muestras para este estudio.

\section{Bibliografía}

BATTARBEE, R. W. 1986. Diatom Analysis. In: BERGLUND, B. E. (ed.), Handbook of Holocene Palaeoecology and Palaeohydrology, pp. 527-570. J. Wiley \& Sons Ltd., New York.

BATTARBEE, R. W. \& M. KNEEN. 1982. The use of electronically counted microspheres in absolute diatom analysis. Limnol. Oceanog. 27: 184-188.

BAHLS, L. 2011. Stauroneis vandevijveri. In: Diatoms of the United States. Retrieved April 14, 2015, from http://westerndiatoms.colorado.edu/taxa/ species/stauroneis_vandevijveri.

BLANCO, S., L. ECTOR \& E. BECARES. 2004. Epiphytic diatoms as water quality indicators in Spanish shallow lakes. Vie Milieu. 54: 71-79.

BLANCO, S., C. CEJUDO-FIGUEIRAS, L. ÁLVAREZBLANCO, E. BÉCARES, L. HOFFMANN \& L. ECTOR. 2010. Atlas de las diatomeas de la Cuenca del Duero. Área de Publicaciones. Universidad de León, León.

COSTE, M. \& L. ECTOR. 2000. Diatomées invasives exotiques ou rares en France: principales observations effectuées au cours des dernières décennies. Syst. \& Geogr. Pl. 70: 373-400.

COX, E. 2003. Placoneis Mereschkowsky (Bacillariophyta) revisited: resolution of several typification and nomenclatural problems, including the generitype. Bot. J. Linean. Soc. 141: 53-83.

ECHAZÚ, D. M. 2012. Biodiversidad de diatomeas en humedales del sur de la Provincia de Santa Cruz, Argentina. Tesis Doctoral, Universidad de Buenos Aires.

FRENGUELLI, J. 1924. Resultados de la Primera Expedición a Tierra del Fuego (1921) - Diatomeas de Tierra de Fuego. Anales Soc. Cient. Argent. 98: 5-63.

FRENGUELLI, J. 1942. Diatomeas del Neuquén (Patagonia). Revista Mus. La Plata, Secc. Bot. 5: 73-219.

FRENGUELLI, J. \& H. A. ORLANDO. 1956. Diatomeas y Silicoflagelados del Sector Antártico Sudamericano. Publ. Inst. Ant. Arg. 5: 1-191.

HLÚBIKOVÁ, D., L. ECTOR \& L. HOFFMANN. 2011. Examination of the type material of some diatom species related to Achnanthidium 


\section{L. García y N. I. Maidana - Diatomeas subfósiles del Brazo Blest, Argentina}

minutissimum (Kütz.) Czarn. (Bacillariophyceae). Algol. Stud. 136/137: 19-43.

IZAGUIRRE, I., P. DEL GIORGIO, I. O'FARRELL \& G. TELL. 1990. Clasificación de 20 cuerpos de agua andino-patagónicos (Argentina) en base a la estructura del fitoplancton estival. Cryptogamie Algol. 11: 31-46.

IZAGUIRRE, I \& J. F. SAAD. 2014. Phytoplankton from natural water bodies of the Patagonian Plateau. Advanc. Limnol. 65: 309-319.

KOCIOLEK, J. P. \& S. A. SPAULDING. 2000. Freshwater diatom biogeography. Nova Hedwigia 71: 223-241.

KRAMMER, K. 1997a. Die Cymbelloiden Diatomeen. Eine Monographie der werltweit bekannten Taxa Teil 1. Allgemeines und Encyonema Part. Bibl. Diatomol. 36, pp. 382. J. Cramer, Berlin - Stuttgart.

KRAMMER, K. 1997b. Die Cymbelloiden Diatomeen. Eine Monographie der werltweit bekannten Taxa Teil 2. Encyonema part., Encyonopsis and Cymbellopsis. Bibl. Diatomol. 37, pp. 470. J. Cramer, Berlin Stuttgart.

KRAMMER, K. 2000. The genus Pinnularia. In: LANGE-BERTALOT, H. (ed.), Diatoms of Europe 1, pp. 703. A.R.G. Gantner Verlag K. G., Ruggell.

KRAMMER, K. \&, H. LANGE-BERTALOT. 1986. Bacillariophyceae 1. Teil: Naviculaceae. In: ETTL, H., J. GERLOFF, H. HEYNIG \& D. MOLLENHAUER (eds.), Süsswasserflora von Mitteleuropa 2, pp. 876. G. Fischer, Jena.

KRAMMER, K. \& H. LANGE-BERTALOT. 1991. Bacillariophyceae 3. Centrales, Fragilariaceae, Eunotiaceae. In: ETTL, H., J. GERLOFF, H. HEYNIG \& D. MOLLENHAUER (eds.), Süsswasserflora von Mitteleuropa 2, pp. 576. G. Fischer, Jena.

KRAMMER, K. \& H. LANGE-BERTALOT. 2004. Bacillariophyceae 4. Teil: Achnanthaceae, Kritische Erganzungen zu Navicula (Lineolatae), Gomphonema Gesamtliteraturverzeichnis Teil 1-4 [second revised edition]. In: ETTL, H., J. GERLOFF, H. HEYNIG \& D. MOLLENHAUER (eds.), Süsswasserflora von Mitteleuropa, pp. 468. Spektrum Akademischer Verlag Heidelberg, Berlin.

KRASSKE, J. 1949. Subfossile diatomeen aus den mooren Patagoniens und Feuerlands. Ann. Acad. Sci. Fenn. IV. Biologica 14: 1-92.

LANGE-BERTALOT, H. 2001. Navicula sensu stricto. 10 Genera separated from Navicula sensu lato. Frustulia. In: LANGE-BERTALOT, H. (ed.), Diatoms of Europe 2, pp. 526. A.R.G. Gantner Verlag K. G., Ruggell.

LANGE-BERTATOLT, H \& G. MOSER. 1994. Brachysira, Monographie der Gattug. Biblioth. Diatomol. 9: 650-669.
LOTTER, A. F., R. PIENITZ \& R. SCHMIDT. 1999. Diatoms as indicators of environmental change near arctic and alpine treeline. In: STOERMER, E. F. \& J. P. SMOL (eds.), The diatoms: Applications for the Environmental and Earth Sciences, pp. 205-226. Cambridge University Press, Cambridge.

LUCHINI, L \& C. A. VERONA. 1972. Catálogo de las diatomeas argentinas. I. Diatomeas de aguas continentales (incluido el Sector Antártico). Comisión de Investigaciones Científicas de la Provincia de Buenos Aires. Monografías 2, La Plata.

MANN, D.G. 1999. The species concept in diatoms. Phycologia 38: 437-495.

MAIDANA, N. I. 1996. Diatomeas fósiles nuevas o poco conocidas para la Argentina: Lago Nahuel Huapi (Brazo Campanario), Prov. de Río Negro. Bol. Soc. Argent. Bot. 31: 177-199.

MAIDANA, N. I. \& C. AMENABAR. 2014. Algae in paleolimnological studies in Argentina. Advanc. Limnol. 65: 323-339.

MAIDANA, N. I, I. IZAGUIRRE, A. VINOCUR, G. MATALONI \& H. PIZARRO. 2005. Diatomeas en una transecta patagónico-antártica. Ecol. Austral 15: 159-176.

MONTOYA-MORENO, Y., S. SALA, A. VOUILLOUD, N. AGUIRRE \& Y. PLATA. 2013. Lista de las diatomeas de ambientes continentales de Colombia. Biota Colomb. 14: 13-78.

MORRONE, J. J. 2001. Homology, biogeography and areas of endemism. Divers. Distrib. 7: 297-300.

PATRICK, R. \& C. W. REIMER, 1966. The diatoms of the United States: Exclusive of Alaska and Hawaii Vol 1. Monographs of The Academy of Natural Sciences of Philadelphia, pp. 688. Philadelphia, Pennsylvania.

RECASENS, C., D. ARIZTEGUI, N. I. MAIDANA, B. ZOLITSCHKA \& THE PASADO SCIENCE TEAM. 2015. Diatoms as indicators of hydrological and climatic changes in Laguna Potrok Aike (Patagonia) since the Late Pleistocene. Palaeogeogr. Palaeoclimatol. Palaeoecol. 417: 309-319.

ROUND, F. E., CRAWFORD, R. M., \& D. G. MANN. 1990. The Diatoms. Biology and morphology of the genera. Cambridge University Press, Cambridge.

RUMRICH, U., H. LANGE-BERTALOT \& M. RUMRICH. 2000. Diatomeen der Anden von Venezuela bis Patagonien/Tierra del Fuego. In: LANGE-BERTALOT, H. (ed.), Iconogr. Diatomol 9, pp. 1-673. A.R.G. Gantner Verlag K.G., Ruggell.

SALA, S. E., J. J. RAMÍREZ \& Y. PLATA. 2008. Diatoms from benthic and lotic systems in Antioquia, Chacó and Santander Departments in Colombia. Rev. Biol. Trop. 56: 1159-1178.

SMOL, J. P. \& B. F CUMMING. 2000. Tracking longterm changes in climate using algal indicators in lake sediments. J. Phycol. 36: 986-1011. 
SPAULDING, S. \& D. M. MC KNIGHT. 1999. Diatoms indicators of environmental change in Antarctic freshwaters. In: STOERMER E. F. \& J. P. SMOL (eds.). The diatoms applications for the environmental and earth science, pp. 245-263. Cambridge University Press, Cambridge.

STRAUB, F. 2002. Note algologique II. Apparition envahissante de la diatomée Achnanthes catenata Bily \& M arvan (Heterokontophyta, Bacillariophyceae) dans le lac de Neuchâtel. Bull. Soc. Neuchât. Sc. Nat. 125: 59-66.

TELL, G. 1985. Catálogo de las algas de agua dulce de la República Argentina. Biblioth. Phycol. 70: 1-283.

TELL, G., I. IZAGUIRRE \& L. ALLENDE. 2011. Diversity and geographic distribution of Chlorococcales (Chlorophyceae) in contrasting lakes along a latitudinal transect in Argentinean Patagonia. Biodivers. Conserv. 20: 703-727.

THOMASSON, K. 1959. Nahuel Huapi. Plankton of some lakes in the Argentine National Park, with notes on terrestrial vegetation. Acta Phytogeogr. Suec. 42: 1-83.

THOMASSON, K. 1963. Araucanian Lakes. Plankton studies in North Patagonia with notes on terrestrial vegetation. Acta Phytogeogr. Suec. 47: 73-103.

URREA, G. \& S. SABATER. 2009. Epilithic diatom assemblages and their relationship to environmental characteristics in an agricultural watershed. Ecol. Indic. 9: 693-703.

VAN de VIJVER, B., Y. FRENOT \& L. BEYENS. 2002. Freshwater diatoms from Ile de la Possession (Crozet Archipelago, Subantarctica). Biblioth. Diatomol. 46, pp. 412. J. Cramer, Stuttgart.

VAN de VIJVER, B., L. BEYENS, \& H. LANGEBERTALOT. 2004. The genus Stauroneis in Artic and Antartic locations. Biblioth. Diatomol. 50, pp. 311. J. Cramer, Stuttgart.

VAN de VIJVER, B., M. KELLY, S. BLANCO, A. JARLMAN, \& L. ECTOR. 2008. The unmasking of a Sub-Antartic endemic: Psammothidium abundans (Manguin) Bukhtiyarova et Round in european rivers. Diatom Res. 23: 233-242.

VAN de VIJVER, B. \& H. LANGE-BERTALOT. 2009. New and interesting Navicula taxa from western and northern Europe. Diatom Res. 24: 415-429.

VANLANDINGHAM, S. L. 1967-1979. Catalogue of the fossil and recent genera and species of Diatoms and their synonyms. I-VIII. J. Cramer, Stuttgart.

VANORMELINGEN, P., E. VERLEYEN \& W. VYVERMAN. 2008. The diversity and distribution of diatoms: from cosmopolitanism to narrow endemism. Biodivers. Conserv. 17: 393-405.

VOUILloud, A. 2003. Catálogo de diatomeas continentales y marinas de Argentina. Versión 1.0. En soporte magnético. Asociación Argentina de Ficología, La Plata.

WERUM, M. \& H. LANGE-BERTALOT. 2004. Diatoms in springs from Central Europe and elsewhere under the influence of hydrogeology and anthropogenic impacts. In: LANGE-BERTALOT, H. (ed.), Iconogr. Diatomol. 13, pp. 479. A.R.G. Gantner Verlag K.G., Ruggell.

WOLFE, A. P. \& H. J. KLING. 2001. A consideration of some North American soft-water Brachysira taxa and description of $B$. arctoborealis sp. nov. LangeBertalot-Festschrift Studies on Diatoms, Dedicated to Dr. Dr. h.c. Horst Lange-Bertalot on the occasion of his 65 th birthday, pp. 633. A.R.G. Gantner Verlag K.G., Ruggell.

ZIDAROVA, R., K. KOPALOVA \& B. VAN DE VIJVER. 2014. The genus Stauroneis (Bacillariophyta) from the South Shetland Islands and James Ross Island (Antarctica). Fottea 14: 201-207.

Recibido el 9 de marzo de 2015, aceptado el 21 de abril de 2015 . 